\title{
NEUES ZU DEN NUTZUNGSPHASEN DES MONUMENTALGRABES VON ANCH-HOR, OBERSTHOFMEISTER DER GOTTESGEMAHLIN NITOKRIS (TT 414)
}

Von Julia Budka

\section{EINLEITUNG}

Die monumentalen Tempelgräber der 25. und 26. Dynastie im Asasif, die für die höchsten Beamten dieser Zeit - in erster Linie für Bürgermeister und Obersthofmeister der Gottesgemahlinnen errichtet wurden, gehören in Theben zu den bekanntesten Monumenten der Spätzeit. In den 1960 er und 1970er Jahren fand eine erste intensive archäologische Erforschung dieses Areals im Vorfeld von Deir el-Bahari statt, die aber nicht zum Abschluss gebracht wurde und mit laufenden Arbeiten der Gegenwart (z.B. in den Anlagen des Padineith, Monthemhat oder Harwa) ihre Fortsetzung findet. ${ }^{1}$

An den frühen Aktivitäten beteiligte sich auch das Österreichische Archäologische Institut in Zusammenarbeit mit dem Institut für Ägyptologie der Universität Wien. Bei den Grabungen unter der Leitung von Manfred Bietak wurden von 1969-1977 in erster Linie Schachtgräber, kleine Grabbauten und eine monumentale Anlage, das Grab des Anch-Hor, Obersthofmeister der Nitokris (TT 414), ausgegraben (Fig. 1). ${ }^{2}$ TT 414 (Fig. 2), das heute für Touristen zugänglich ist, wurde 1978 und 1982 als zweibändige Monographie publiziert, in der neben dem Bau- und Grabungsbefund die in situ dokumentierten Funde vorgelegt wurden. ${ }^{3}$ Die Masse der Funde ist jedoch bislang unveröffentlicht und wurde im provisorischen Magazin der Grabung vor Ort belassen (Saffgrab Nr. 1, s. u.).

Ähnlich wie mit TT 414 verhält es sich mit

$1 \mathrm{Zu}$ diesen aktuellen Arbeiten s. beispielsweise GomaÀ 2004; GOMAà 2006; TIRADRITTI 2004a; TIRADRITTI 2004b; TiradritTI 2004c; TIRADRITTI 2004d; TIRADRITTI 2004e; TIRADRITTI 2005a; TIRADRITTI 2005b; TIRADRITTI 2005c; TIRADRITTI 2006.

2 S. BiETAK 1972; BIETAK 1973; BIETAK 1976-1977; BIETAK 1974-1977; BUDKA 2006.

3 BietaK \& Reiser-Haslauer 1978; BietaK \& Reiser-HasLAUER 1982.

4 Vgl. z.B. Assmann 1973; Assmann 1977; Eigner 1984; Graefe 1990; KuHlmann \& Schenkel 1983.

gypten und Levante/Egypt and the Levant 18, 2008, 61-85

Ägypten und Levante/Egypt and the der Wissenschaften, Wien anderen Tempelgräbern im Asasif: Die aufwendige Architektur der Grabbauten, ihr Bild- und Textprogramm, die allesamt sowohl Innovationen als auch Rückgriffe auf verschiedene Epochen erkennen lassen, waren bereits Gegenstand mehrerer Studien, ${ }^{4}$ während das zugehörige Fundmaterial, das meist sehr fragmentarisch erhalten ist, kaum bekannt oder durch Publikationen zugänglich ist. ${ }^{5}$ An diesem Punkt, einem noch unzureichenden Verständnis der Bestattungspraktiken und Grabinventare der thebanischen Monumentalgräber der Spätzeit, setzt ein neues Forschungsprojekt des Österreichischen Archäologischen Instituts, Zweigstelle Kairo mit Beteiligung der Humboldt-Universität zu Berlin an.

\section{Die AUfarbeitungSKaMPagnen 2007-2008}

Während der österreichischen Ausgrabungen in den 1960ern und 1970ern diente das geräumige Saffgrab „Grab I“ des Konzessionsgebiets als Magazin für das Fundmaterial. Nach Abschluss der Arbeiten gelangten die besterhaltenen Stücke durch Fundteilung nach Kairo (Ägyptisches Museum) sowie nach Wien (Kunsthistorisches Museum). Ein Großteil des Materials, v.a. Keramik und fragmentierte Stücke der Grabinventare, verblieb hingegen vor Ort in Grab I. Dieses Magazin wurde 2002 geöffnet und die Objekte, die aus den nicht-monumentalen Anlagen des Areals stammen, konnten bearbeitet werden. ${ }^{6}$ Diese Analyse erbrachte wesentliche neue Erkenntnisse zum spätzeitlichen Bestat-

\footnotetext{
Die positive Ausnahme stellt in dieser Hinsicht das Grab des Padihorresnet dar, aus dem ein großer Teil des Fundmaterials vorgelegt wurde, s. GRAFFE 2003. Bedauerlicherweise wurde bei diesem Korpus aber weder versucht, das Material zu kontextualisieren noch genau zu datieren oder in Hinblick auf die Nutzung des Grabes zu interpretieren; die Funde, besonders die Keramik, wurden hingegen in knapper Katalogform publiziert.

BUDKa 2006, Bd. IV, 3-6.
} 


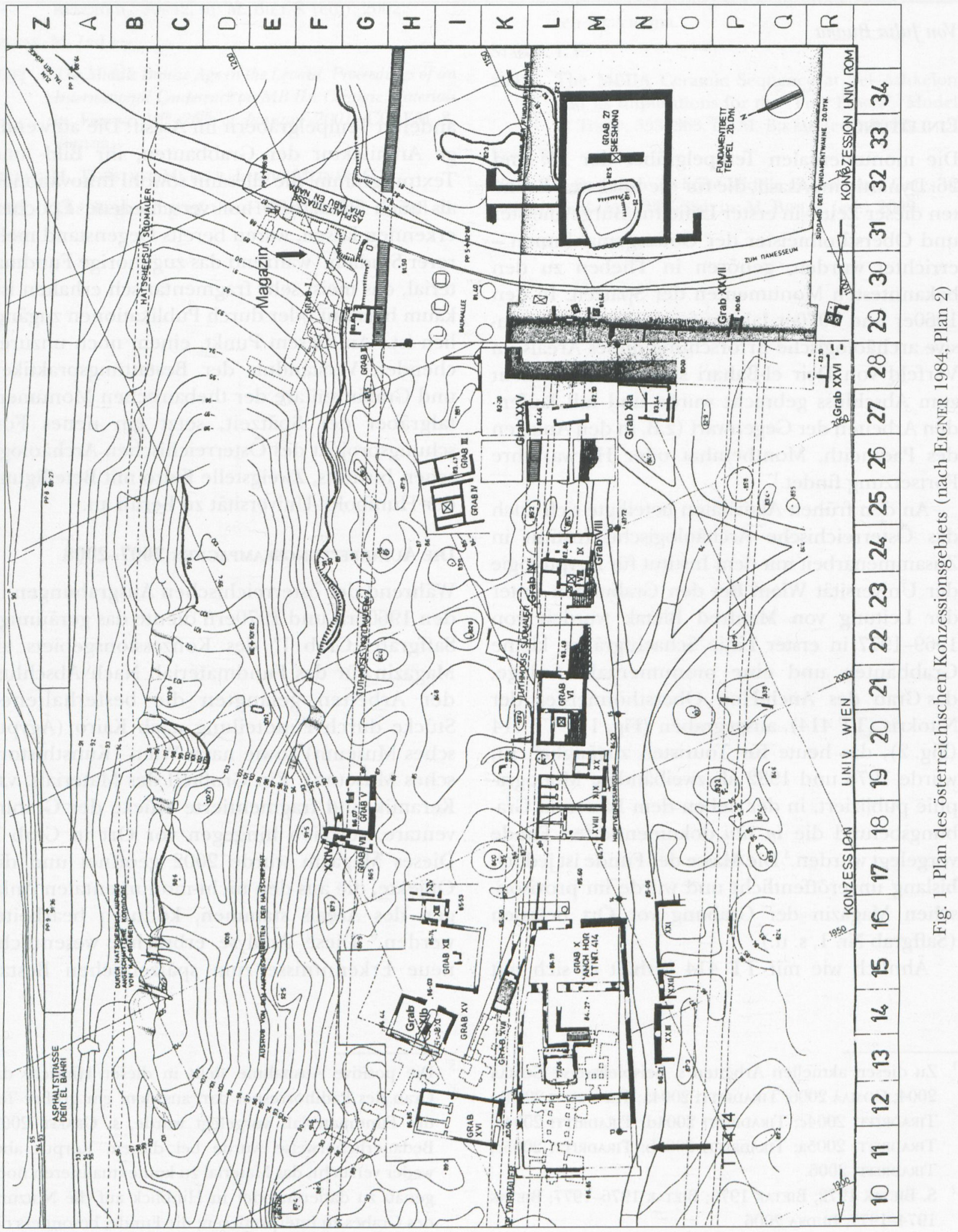




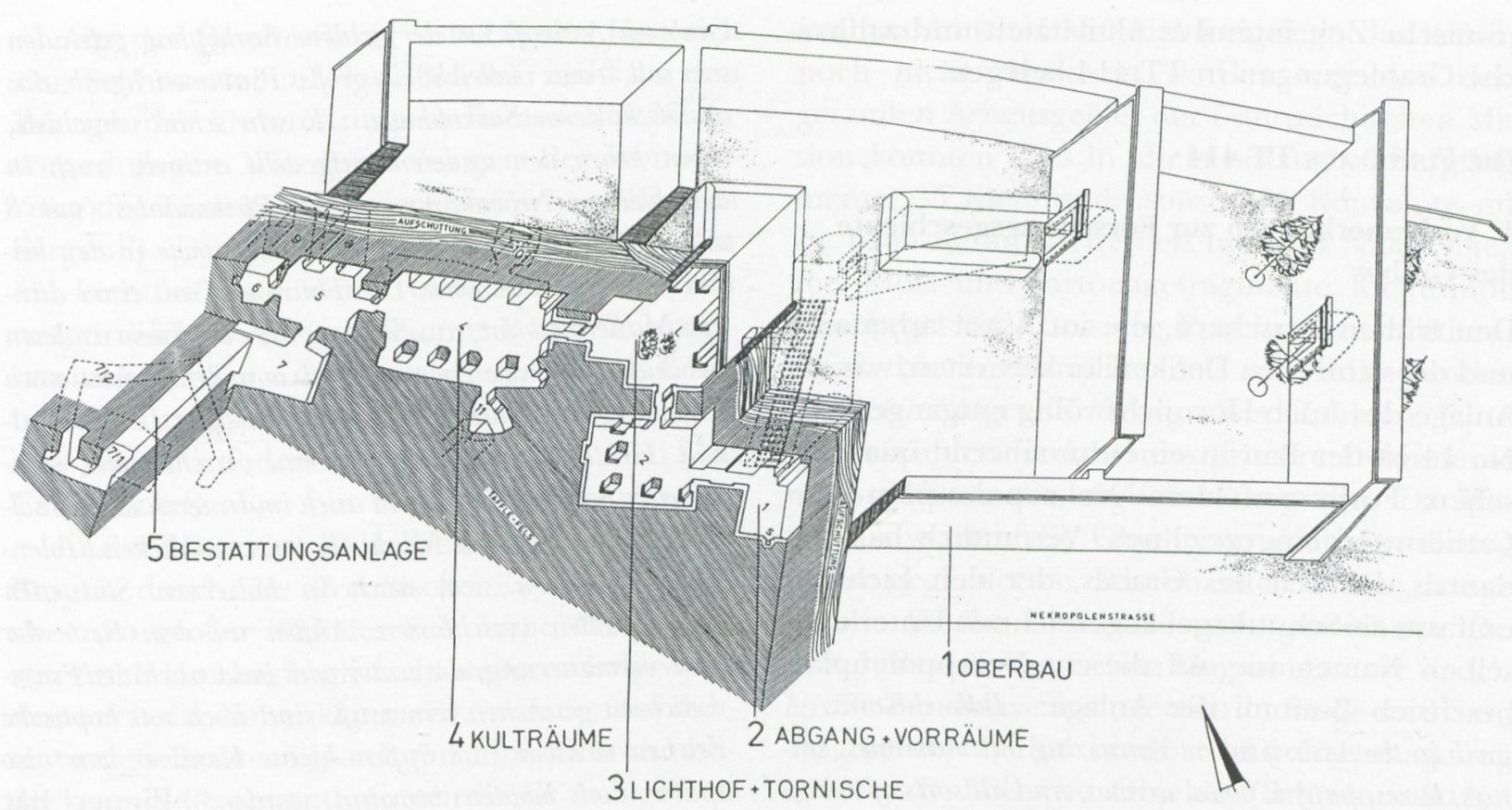

Fig. 2 Isometrischer Schnitt durch das Grab des Anch-Hor (aus: EIGNER 1984, Abb. 113)

tungswesen und Kultvollzug in Gräbern von Personen mittlerer und niedriger Ränge im Umfeld der monumentalen Tempelgräber der höchsten Beamten. Neben der Rekonstruktion von Grabinventaren aus der 25. und 26. Dynastie ergab sich das Bild einer sehr intensiven Wiederbenutzung des gesamten Areals und der einzelnen Grabbauten als Kult- und Bestattungsplätze im 4. und 3. Jahrhundert v. Chr. ${ }^{7}$ Die so rekonstruierbaren zwei Hauptnutzungsphasen des Asasif, zum ersten während der Kuschiten- und Saitenzeit und zum zweiten während der 30. Dynastie sowie der frühptolemäischen Zeit, sind sowohl in den kleinen als auch den monumentalen

\footnotetext{
S. BuDKa 2006, bes. Bd. III, 784-815.
}

8 Die Teilnehmer der Kampagnen waren: Manfred Bietak (Ägyptologe, Projektleiter); Julia Budka (Ägyptologin, Leitung vor Ort); Sara Bock, Jana Helmbold, Veronika Hinterhuber (Ägyptologinnen, Humboldt-Universität zu Berlin); Arvi Korhonen (Student, Humboldt-Universität zu Berlin); Erico Peintner \& Ahmed Refaat Eisa Abo El-Ata (Restauratoren, Kairo); Zsuzsanna Thót (Ägyptologin/Archäologin, Universität Budapest); Katharina Vogt (Studentin, Humboldt-Universität zu Berlin) und Angelika Zdiarsky (Ägyptologin, Universität Wien). Als Inspektoren des ägyptischen Antikendienstes unterstützten uns Ahmed Hassan Abeed (2007), Hassan Ramadan Mahmoud (2007) und Abdel Hakiem Ahmed
Spätzeitgräbern zu fassen, wobei in der Regel die Befunde aus der zweiten Blütezeit (4./3. Jahrhundert v. Chr.) besser erhalten sind. Auf diesen Ergebnissen zu den Grabinventaren und mehrfachen Nutzungsphasen der spätzeitlichen Nekropole in Theben bauen die aktuellen Arbeiten in der österreichischen Konzession auf. Seit 2007 stehen die Objekte aus dem Monumentalgrab des Anch-Hor (TT 414) im Fokus. Die beiden jüngsten Aufarbeitungskampagnen, die vom 1. Oktober bis 25. November 2007 und vom 13. Februar bis 13. März 2008 im Magazin stattfanden, ${ }^{8}$ konzentrierten sich auf Keramik, Särge und Holzobjekte, die von der 26. Dynastie bis in

el-Sorghiar (2008). Für die Arbeitserlaubnis sind wir Dr. Zahi Hawass und dem Permanent Committee des SCA zu Dank verpflichtet. Ohne die aktive Unterstützung und Zusammenarbeit mit den lokalen Behörden in Luxor wäre das Projekt nicht durchführbar gewesen. Wir danken besonders Dr. Mansour Bourraik, Generaldirektor des Südlichen Oberägyptens; Ali el-Asfar, Generaldirektor der Westseite Thebens; Nour Abdel Ghaffar, Direktor der Westseite; Fathy Yasin, Chefinspektor der Westseite und El-Kazafy Abd el-Raheem, Chefinspektor für Magazine und Museen. Die Arbeiten wurden durch finanzielle Unterstützung der Gerda Henkel Stiftung, der Humboldt-Universität zu Berlin und dem Österreichischen Archäologischen Institut Kairo ermöglicht. 
römische Zeit intensive Aktivitäten und zahlreiche Grablegungen in TT 414 belegen.

\section{Die Funde Aus TT 414}

\section{Vorbemerkungen zur Forschungsgeschichte des Grabes}

Den frühen Forschern, die im Asasif arbeiteten und die sichtbaren Denkmäler kartierten, war die Anlage des Anch-Hor nicht völlig entgangen. Als Nr. 14 ist der Bau in einer annähernd quadratischen Form auf dem Nekropolenplan von Catherwood eingezeichnet. ${ }^{9}$ Vermutlich hat sich damals der Teil des Grabes, der den Lichthof umfasst, als Schuttkegel abgezeichnet. Unter dem selben Namen wie auf diesem Nekropolenplan beschrieb Bonomi die Anlage: „Bab el-Goria: A tomb in the Assasif where Yanni dug out mummies, statues, boxes, papyri, bows, arrows, and all sorts of things found in tombs. "10 Die große Fülle an Gegenständen, die aus dem Bau geborgen wurden, erwähnte auch noch etwas später Lane (,great quantity and variety of antiques"11). Ein Teil dieser in den 1810ern und 1820ern zutage gekommenen Funde wurde über die Sammlung von Henry Salt von London (British Museum) und Turin (Museo Egizio) erworben. Es handelt sich vorwiegend um Stelen, Uschebtis und Totenbuchpapyri, die Belzoni zwischen 1817 und 1819 für Salt beschafft hatte. ${ }^{12}$ Uschebtis des Anch-Hor haben in der ersten Hälfte des 19. Jahrhunderts mehrere europäische Sammlungen erstanden (z.B. das Rijksmuseum Leiden, der Louvre und das Ashmolean Museum in Oxford). ${ }^{13}$

Eine große Zahl von Särgen sah noch Richard Lepsius im Grab aufgestellt. Lepsius scheint, wie seine Vorgänger, über den Lichthof in die Anlage gekommen zu sein, drang aber bereits bis in den westlichen Teil der unterirdischen Kultanlage, die große Pfeilerhalle, vor. Er beschrieb seinen Eindruck wie folgt (LD, Text III, 284 $\left.{ }^{14}\right)$ : „Das große
Grab im Assasif, wo der goldene Sarkophag gefunden sein soll, ${ }^{15}$ war vielleicht ein großes Psammetichgrab, das an 30 hölzerne Sarkophage, z.T. sehr schön vergoldete, einen neben den anderen aufgestellt enthielt; auch in den kleinen Nebenkammern des Pfeilersaales (mit 8 rohen Pfeilern und 8 rohen Wänden) sowie in den beiden hinteren Kammern. Die Thüre war mit einer dünnen Mauer verbaut, aus Steinen, die von einem anderen Gebäude genommen waren und von denen jetzt noch einer umgekehrt die Schilder Psammetichs II. (...) enthält. Hinter dieser Mauer war eine verschlossene, zweiflügelige hölzerne Thür und auch im Inneren noch 2 solche Thüren. Fast jede Mumie hatte einen dicken (Toten)Papyrus neben sich, auch in hölzernen Statuetten waren Rollen verschlossen. Außen an dem Reste der Thürvermauerung, die unter oder bald nach der Psammetichzeit geschehen sein muß, sind noch rote koptische Zeichen sichtbar; die äußere kleine Kammer war also später von Kopten bewohnt worden." Eigner hat bereits darauf hingewiesen, dass es sich bei der Schilderung der Thüre nur um die Tornische vom Lichthof zur Pfeilerhalle (Raum 4 auf Abb. 2) handeln kann. ${ }^{16}$ Folgerichtig muss mit „äußere kleine Kammer" der Lichthof des Grabes ( $\mathrm{L}$ auf Abb. 2) gemeint sein. Drei Punkte scheinen mir hier bei der Beschreibung der Architektur wesentlich: 1) Lepsius kannte die reale Eingangssituation des Grabes nicht; er verschaffte sich Zutritt über den Lichthof, der nach oben offen war und durch anstehende Schuttmengen oder Einbauten sehr klein wirkte. 2) Der hintere Abschnitt der unterirdischen Kultanlage, beginnend mit der 8-Pfeilerhalle (Raum 4), war damals noch vermauert gewesen. Anders als Lepsius vermutet, sollten diese Abmauerung und die Holztüren aus römischer Zeit stammen. ${ }^{17}$ 3) Es scheint so, als wären beim Besuch durch Lepsius die hinteren Nebenkammern (Räume 7,8 und 9) noch nicht sichtbar gewesen; vermutlich meinte er mit „die beiden hinteren Kammern" die Räume 5 und 6 auf dem durch Bietak publizierten Plan (Fig. 2).

\footnotetext{
9 S. dazu Eigner 1984, 54 sowie Abb. 4.

10 Bonomi 1906, 81, Nr. 23; s. auch Eigner 1984, 54.

11 LANE 2000, 333.

12 S. Bierbrier 1987, 23, 30, 36-39, bes. 37; Quirke 1993, 6, 12 und 21 sowie Munro 1973, 57-59. Zu diesen intrusiven Bestattungen und ihren Zeugnissen vgl. auch de Meulenaere 1984, 238-241; de Meulenaere 1989, 63-69.
} 
Aus der Beschreibung von Lepsius lässt sich der Schluss ziehen, dass Belzoni nicht in den Bereich der hinteren unterirdischen Kultanlage vorgedrungen sein kann (wegen der Abmauerung der Türe und der noch aufrecht stehenden Särgen $\left.{ }^{18}\right)$. Das reiche Fundgut scheint vielmehr im Lichthof und dem vorderen Bereich der unterirdischen Anlage (Räume 1 und 2) aufgesammelt worden zu sein, wobei der Hof als Kernbau des Grabes aufgefasst wurde (s. die quadratische Form auf dem oben erwähnten Plan von Catherwood).

In der Mitte des 19. Jahrhunderts, nach dem Besuch durch die Preußische Expedition, war aber ein großer Teil der Felsräume des Grabes von Anch-Hor zugänglich. In weiterer Folge wurden auch die Nebenkammern, in denen Schächte zu den Bestattungsanlagen hinabführen, entdeckt und die gesamte unterirdische Anlage von Plünderern durchsucht. ${ }^{19}$ Zur Wiederentdekkung der Anlage, ihrer vollständigen systematischen Ausgrabung und der Identifizierung des Grabbesitzers sollte es aber erst in den 1970er Jahren kommen. Die Größe des Grabes, seine lange Belegungsgeschichte und die Vielzahl der Bestattungen spiegelt sich in der Fülle des Materials wider, die durch die Ausgrabungen der österreichischen Mission trotz der mehrfachen antiken Beraubung, der im 19. Jahrhundert durchgeführten partiellen Freilegung und der damit verbundenen sukzessiven Aufsammlung von beweglichem (und „lohnenswertem“) Inventar noch zutage kam.

\section{Die Magazinbestände in Theben}

Zentrales Arbeitsziel der beiden jüngsten Kampagnen war es, den gesamten Inhalt des Magazins zu erfassen. Dabei wurden etliche bereits während der Grabung registrierte Gegenstände wiederentdeckt - 212 einzelne Särge und Sargfragmente, 150 kleine Einzelobjekte (Mumienbinden, Statuetten und Fragmente von solchen, Uschebtis und Amulette) und 200 Fundkonvolute (Holzund Kartonagefragmente, Kleinfunde aus Fayence und Stein, Tonobjekte und Keramik) -, aber auch zahlreiches Material freigelegt, das bislang noch nicht registriert worden war. Aus dem gesamten Arbeitsgebiet der österreichischen Mission konnten alles in allem bislang 60 Sargfragmente, 35 Kleinfunde sowie 535 Konvolute mit ca. 6200 Keramikscherben und fast 6500 Objekten (Holz- und Kartonagefragmente, Kleinfunde, Grabkegel etc.) aus der Epoche vom Mittleren Reich bis in Römische Zeit neu dokumentiert werden. Aufgrund der großen Zahl an Fundgegenständen ist dabei das primäre Dokumentationsmedium die digitale Fotografie. Darüber hinaus werden die Objekte in nach Fundkategorien gegliederten Datenbanken erfasst, ausgewählte Stücke zusätzlich auch gezeichnet. Der Inhalt des provisorischen Magazins wurde in acht große Fundklassen eingeteilt: 1) Särge und Sargfragmente, 2) Kartonagen und Kartonagefragmente, 3) hölzerne Objekte (Schreine, Stelen, Statuetten etc.), 4) Steinobjekte und -blöcke (v.a. Opferplatten und Reliefsteine), 5) Kleinfunde (Uschebtis, Amulette, Grabkegel, Tonverschlüsse etc.), 6) Keramik (vollständige Gefäße und Scherben), 7) gemischte Konvolute (Holz- und Kartonagereste, Kleinfunde, Keramik etc.) sowie 8) menschliches Mumien- und Knochenmaterial. Diese Fundgattungen wurden getrennt von einander sortiert und sind jetzt gemäß ihrem Bearbeitungsstand im Magazin gelagert.

Der Großteil der genannten Objekte wurde in TT 414 gefunden. Die allgemeinen Nutzungsphasen dieser Anlage sind bereits vorgelegt worden. ${ }^{20}$ Doch allein die kontextuelle Auswertung des gesamten Fundmaterials, mit Berücksichtigung der umfangreichen Magazinbestände, kann letztlich die einzigen sicheren Ansatzpunkte zur detaillierten Rekonstruktion der Belegung und Nutzungsdauer der Anlage liefern. Diesbezügliche erste Eindrücke werden im Folgenden vorgestellt.

\section{Fundmaterial aus der 26. Dynastie}

Der Befund zur primären Nutzungszeit des Grabes hat stark unter der mehrfachen Wiederbenutzung, den verschiedenen Umbauten und

\footnotetext{
18 Die Särge, die laut der Beschreibung von LEPSIUS noch in der Pfeilerhalle aufrecht standen, können nicht älter als die 30. Dynastie sein. In dieser Epoche wurde die Vergoldung von Gesicht und Brust beliebt und hielt sich über die ptolemäische Periode bis in die Römerzeit.
}

\footnotetext{
19 Bietak \& Reiser-Haslauer 1978, 75 (unentdeckt blieb allein Raum 10.2, s. u.).

20 BietaK \& Reiser-Haslauer 1978; Bietak \& Reiser-HasLAUER 1982.
} 
Plünderungen gelitten. Quantitativ überwiegen deutlich die Funde späterer Nutzungsphasen, v.a. aus der 30. Dynastie und der Ptolemäerzeit. Dennoch konnten bereits Reiser-Hauslauer und Bietak Reste des Grabinventars von Anch-Hor und verschiedener Verwandten identifizieren (z.B. von seiner Tochter Merit-Neith). ${ }^{21}$ Die Uschebtis des Anch-Hor fanden durch die frühen „Ausgrabungen" des 19. Jahrhunderts in verschiedenen Museen Eingang (s. o.); wenige vollständige Exemplare und Fragmente von solchen wurden noch während der österreichischen Grabung in der Bestattungsanlage des Grabes gefunden.

Vor Ort im Asasif sind die Reste des Sargensembles von Anch-Hor geblieben. Das Fußteil Reg. $697^{22}$ belegt einen äußeren, bunt bemalten Sarg, dessen dekorierte Stuckauflage heute leider sehr schlecht erhalten ist. Ursprünglich ließ sich auf dem unteren Abschluss des Deckels die Kartusche der Nitokris innerhalb der Titelreihe des Anch-Hor lesen. ${ }^{23}$ Deckelfragmente des inneren Holzsarges (Reg. 537) ${ }^{24}$ haben sich inklusive der aufgesetzten Gesichtsmaske erhalten. Obgleich auf den ersten Blick unscheinbar, sind diese wenigen Reste aufgrund ihrer sicheren Datierung um 585 v. Chr. von hoher Bedeutung für eine Typologie der Särge dieser Epoche. ${ }^{25}$ Für die spezifische Sargtypologie innerhalb von TT 414 ist das Sargensemble des ursprünglichen Grabbesitzers ebenfalls von Relevanz - so hat es den Anschein, als wären verschiedene Motivelemente und die Farbgebung bis in ptolemäische Zeit nachempfunden worden (s. u.).

Ein Neufund aus dem Jahr 2007 verdient hier hervorgehoben zu werden. Es handelt sich um einen schmalen Holzsplitter mit einer Länge von etwa $70 \mathrm{~cm}$ (K07/327.1, Fig. 3), der den Rest einer geschnitzten, vertikalen Textkolumne zeigt.

${ }^{21}$ S. BietaK \& Reiser-Haslauer 1982, 167-178.

${ }^{22}$ Bietak \& Reiser-Haslauer 1982, 170-175, Abb. 71-73 und Taf. 94-96.

23 Als Titel 12 des Anch-Hor (jmj-r3 pr wr (n) dw3.t-ntr), s. Bietak \& Reiser-Haslauer 1978, 52; zum Sarg s. BietaK \& Reiser-Haslauer 1982, 170.

24 Bietak \& Reiser-Haslauer 1982, 167-170, Abb. 69, 70, 73 und Taf. 93.

25 Zur Bedeutung des Sarges s. TAYlor 2003, 119. Das genaue Datum ergibt sich durch die Amtszeit des Anch-Hor - im Jahr 586 war bereits sein Nachfolger Scheschonk installiert, wie es eine Stele der Anchesneferibre belegt (EIGNER 1984, 16).
Die Hieroglyphen waren ehemals gelb eingelegt. Der Text lässt sich als der Name des Anch-Hor ergänzen, dessen Titelfolge hier die Kartusche der Gottesgemahlin Nitokris enthält, wie es auch am äußeren Sarg Reg. 697 belegt ist (s.o.): [jmj-r3
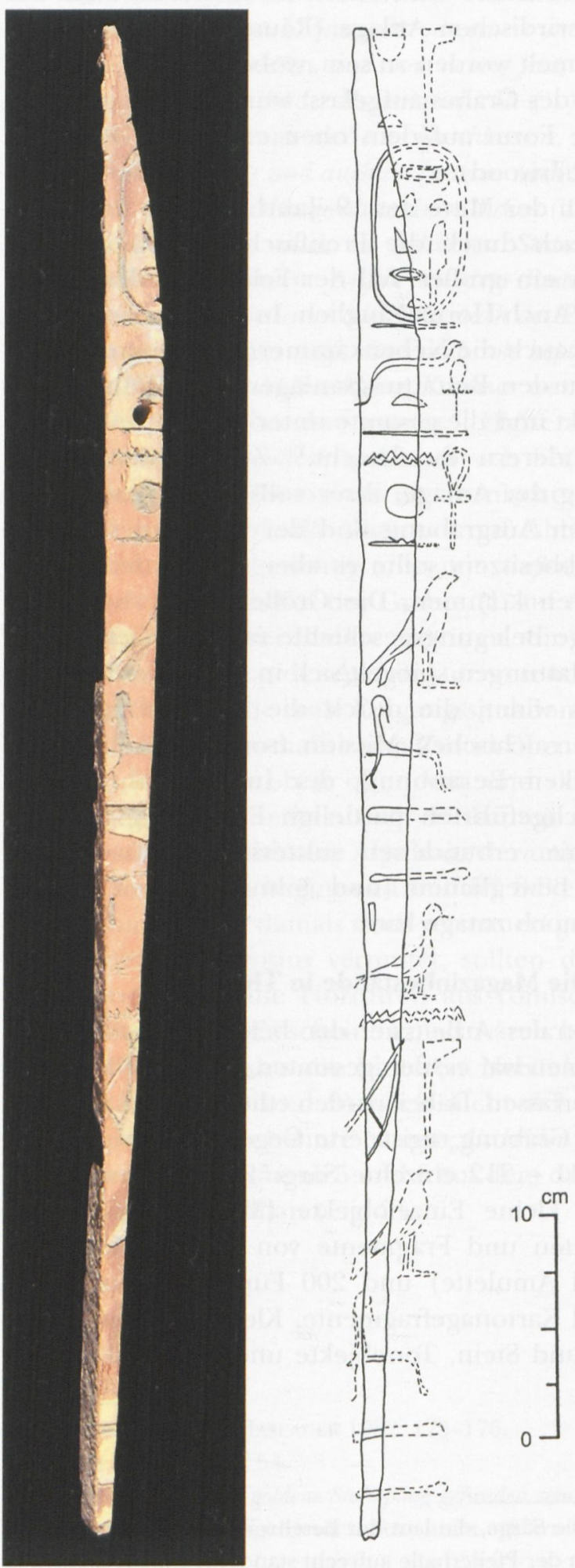

Fig. 3 a) Das Sargfragment K07/327.1 mit dem Namen des Anch-Hor; b) Rekonstruktion der Inschriftkolumne von K07/327.1 (maßgerechte Skizze nach dem Foto; J. Budka) 


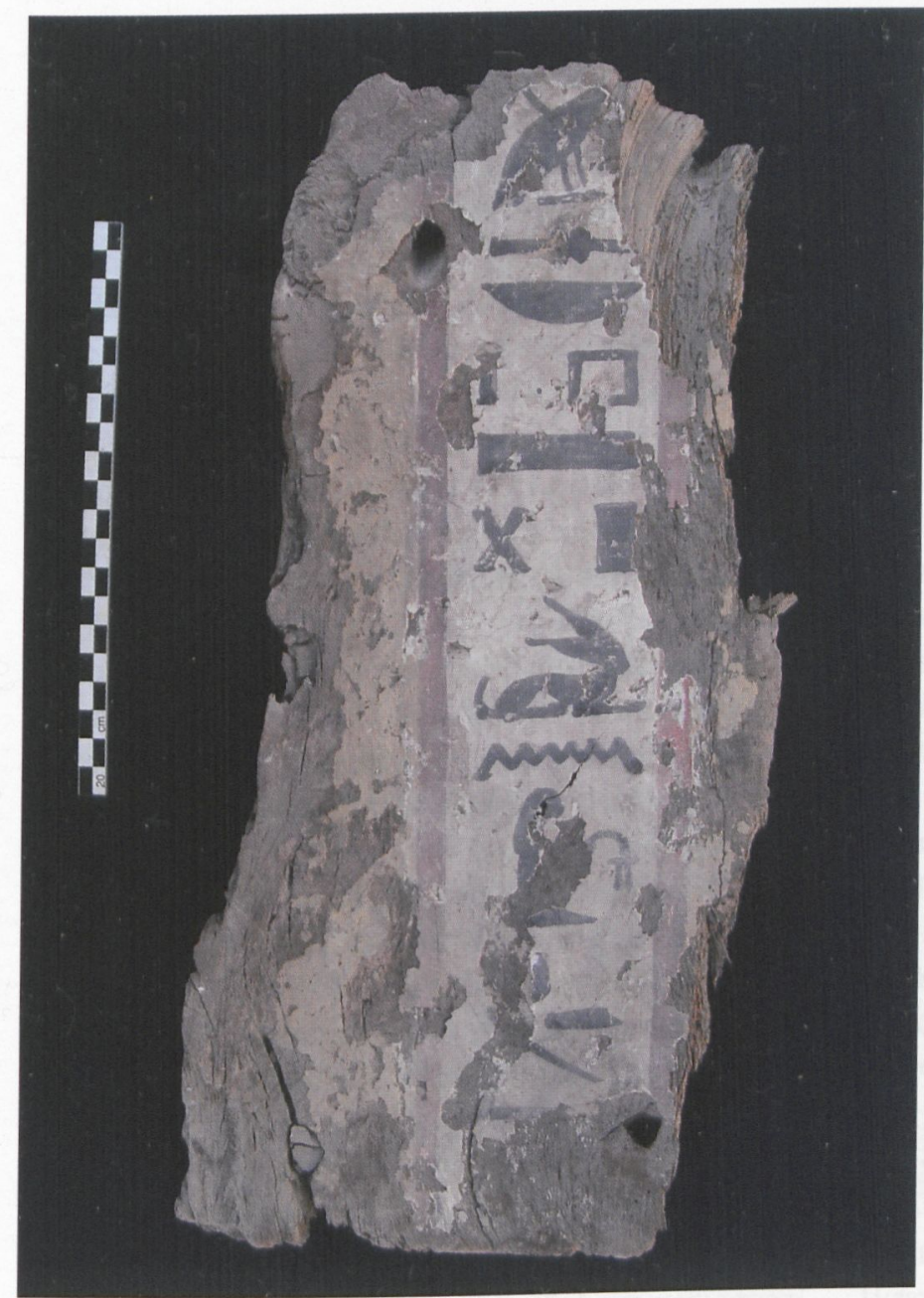

Fig. 4 Das Sargfragment einer Schwester des Anch-Hor (Reg. 868)

Fig. 5 Die fragmentarisch erhaltene Stele Reg. 792 (26. Dynastie)

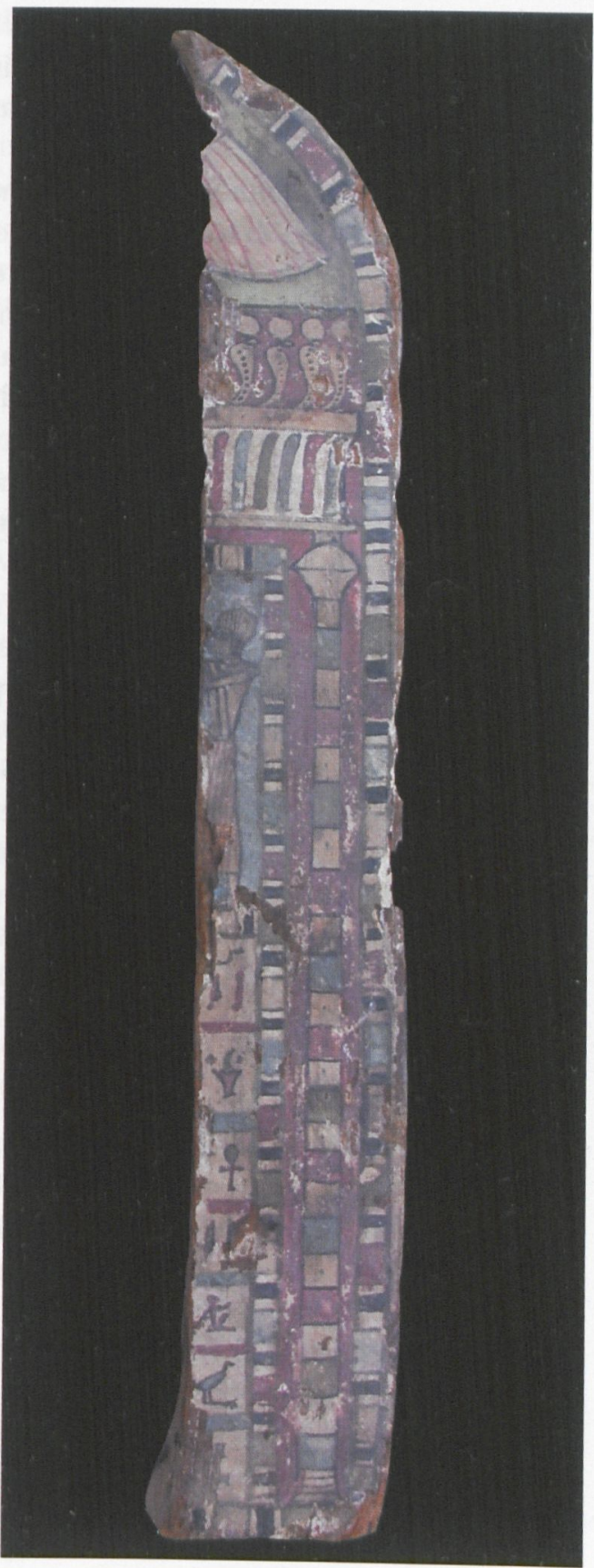

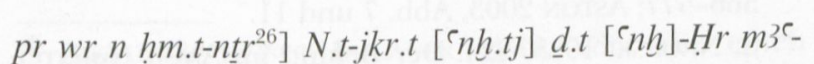
hrw [s3] $n$ mrj-[ntr] [Hr] $m^{3}$-hrw „... der Obersthofmeister der Gottesgemahlin Nitokris - sie lebe ewiglich -, Anch-Hor, der Gerechtfertigte, Sohn des Gotteslieblings ${ }^{27}$ Hor, des Gerechtfertigten..."

26 Als wahrscheinlichere Variante statt dw3.t-ntr rekonstruiert; vgl. BIETAK \& Reiser-Haslauer 1978, 50, E1.

27 Zum Titel (jt-ntr) mrj-ntrr bei Vätern von Obershofmeistern s. GRAEFE 1978, 43 mit weiteren Angaben.

${ }^{28}$ Oder sollte K07/327.1 doch einen mittleren anthropoiden Sarg des Anch-Hor belegen? Dagegen spricht
Da für Anch-Hor aber ein bunt bemalter äußerer und ein ebensolcher innerer Sarg nachgewiesen sind, spricht vieles dafür, dass es sich beim neuen Fragment K07/327 um den Sarg eines Familienmitgliedes handelt. $^{28}$ Möglich wäre

jedoch, dass bei einem Dreier-Sargensemble als äußerer Sarg ein krsw-Sarg zu erwarten wäre. Außerdem ist die Rekonstruktion der Proportionen von innerem und äußerem Sarg sehr plausibel (BIETAK \& REISERHaslauer 1982, Abb. 73) und lässt keinen Platz für einen mittleren Sarg. 
zunächst die Tochter Merit-Neith, die nachweislich in TT 414 bestattet wurde,${ }^{29}$ wobei aber bislang nur Reste eines Haussarges zum Vorschein kamen. Die Gemahlin des Anch-Hor, die ebenfalls in Frage käme, ist bisher namentlich nicht bekannt. ${ }^{30}$ Denkbar ist auch, dass die Mutter des Anch-Hor, Schepen-wen (Šp-n-wn, vgl. Fig. 4), ${ }^{31}$ bei ihrem Sohn bestattet war - hier wäre aber eher ein direkter Bezug auf ihren Gatten (Hor) zu erwarten und nicht dessen Nennung als Vater des Anch-Hor wie bei K07/327.1. Letzteres würde am besten zu einem direkten Nachkommen des Anch-Hor passen - zur oben genannten Tochter oder auch zu einem bislang nicht belegten Sohn. Es bleibt zu hoffen, dass im Magazin noch weitere Fragmente auftauchen, die erklärende Informationen liefern.

Die bereits bekannten Reste von Särgen mehrerer Familienmitglieder des Anch-Hor aus TT $414^{32}$ lassen eine große Bandbreite an Stilen und deutliche Qualitätsunterschiede in ihrer Ausführung erkennen, wofür es noch keine eindeutige Erklärung gibt. ${ }^{33}$ Zwei Fragmente eines bunt bemalten äußeren Sarges belegen die Bestattung einer namentlich nicht bekannten Schwester des Anch-Hor (Reg. 868, Fig. 4). Sehr schlecht erhalten ist leider die äußere Beschriftung der Sargwanne eines Bruders von Anch-Hor, Psmtk-mn-mW3s.t. Der Sarg (Reg. 591) aus der Schachtverfüllung von Raum 10 wurde mit bemalter Leinwand überzogen und dieser Stoff hat sich mittlerweile gelöst. Eine erste Konsolidierung des Stückes, das ebenfalls für die saitische Sargentwicklung in Theben von Belang ist, ${ }^{34}$ konnte 2007 erfolgen und wird sobald als möglich fortgesetzt werden.

Neben Särgen und Uschebtis sind von den

29 Zu den Resten ihres Grabinventars s. BIETAK \& REISERHASLAUER 1982, 176-178.

30 Vgl. Graefe 1978, 42 mit Anm. 159a.

31 BietaK \& Reiser-Haslauer 1982, 281, G 132.

32 S. Bietak \& Reiser-Haslauer 1982, 250-251.

33 Chronologische Faktoren sind ebenso wie veränderte soziale Verhältnisse der Familie denkbar, wobei diese in inhaltlichem Zusammenhang stehen könnten und daher nicht eindeutig voneinander zu trennen sind.

34 Als Vergleichsbeispiel s. z.B. TAYLOR 1989, 59, Abb. 48 (Leiden Inv. AMM 4, sp. 25.-26. Dyn.).

35 S. BietaK \& Reiser-Haslauer 1982, 177-178, Abb. 76; allg. zu Ptah-Sokar-Osiris-Statuetten aus der österreichischen Konzession im Asasif s. BUDKA 2003.

36 S. Bietak \& Reiser-Haslauer 1978, 140.

37 Diese Anahme basiert neben der Materialwahl (empfindlicher Werkstoff Holz) auf gesicherten Befunden
Bestattungen der 26. Dynastie in TT 414 PtahSokar-Osiris-Statuetten ${ }^{35}$ und Stelen belegt. Letztere treten sowohl in Stein als auch in Holz auf. Die Kalksteinstele der Tochter des Anch-Hor, MeritNeith, wurde aus mehreren Fragmenten aus sekundärer Fundlage zusammengesetzt und war vermutlich einst im Lichthof aufgestellt. ${ }^{36}$ Holzstelen gehörten seit der Dritten Zwischenzeit zum beweglichen Grabinventar thebanischer Bestattungen und wurden im Unterschied zu den Exemplaren aus Stein den Bestattungen beigegeben. ${ }^{37}$ Es handelte sich aber wohl um keinen obligatorischen Teil des Grabinventars, was dadurch deutlich wird, dass etwa in der zweiten Cachette von Deir el-Bahari bei 153 Särgen nur 8 Stelen gefunden wurden. ${ }^{38}$ Die Aufstellung von Holzstelen während der Dritten Zwischenzeit bis in die Spätzeit beim Sarg - also in der Grabkammer - stellt eine Neuerung dar, die sich gegen den sonst üblichen, nach außen gerichteten Charakter der Denksteine als Kommunikationsmedien wandte. ${ }^{39}$

Einige Holzstelen der 26. Dynastie kamen bei den österreichischen Grabungen in TT 414 zutage. Eine vollständige Stele, die im Schacht von Raum 10 in 5 m Tiefe der Verfüllung gefunden wurde, befindet sich heute in Wien (Reg. 506, KhM A2096). ${ }^{40}$ Das leicht verzogene Holz des Stückes ist der Länge nach in mehrere Teile gespalten und die Stuckschicht ist teilweise abgeblättert. Die Bemalung wurde auf weißem Grund in rot, blau, grün, schwarz und gelb ausgeführt. Das Stück entspricht dem Typ Munro II Rhg. D (Datierung: 620-580 v. Chr.) und kann aufgrund seines Fundkontextes gegen 580 v. Chr. datiert werden. ${ }^{41}$ Der Name des Besitzers ist zwar nicht

aus der fraglichen Epoche, vgl. Aston 1987, Bd. II, 566-577; Aston 2003, Abb. 7 und 11.

38 S. NiwInSki 1988, 224. Der Befund im Asasif (österreichische Konzession) scheint eine uneinheitliche Verwendung zu bestätigen, denn Belege für Holzstelen fehlen bislang aus den nicht-monumentalen Anlagen und kommen ausschlieBlich aus dem Grab des Anch-Hor.

${ }^{39}$ Zum veränderten Charakter der Stelen in der Dritten Zwischenzeit, wobei noch einige Fragen ungeklärt sind, s. TAYLOR 2001a, 161; LOTH 2008.

40 SAtZinger 1979, 106-107, M 4 (Abb. 95); BudKA 2006, Bd. IV, 191-192, Kat. 497.

41 Gute Parallelen sind Edinburgh 1885.139 (Munro 1973, 221); Wien 891 (Munro 1973, 223, 620-560 v. Chr.); (Philadelphia University of Pennsylvania 2042 (Quibell \& GRIFFITH 1896, Taf. 21, Nr. 15 = LOTH 2008, Abb. 8, 26. Dyn.). 
mehr lesbar, aber aufgrund des Titels „Kammerherr der Gottesverehrerin" (jmj-hnt $n$ dw3.t-ntr) wird es sich um ein Familienmitglied des Anch-Hor gehandelt haben, ${ }^{42}$ das vielleicht in der Grabkammer 10.1 bestattet gewesen ist, wo vermutlich auch die Stele deponiert wurde.

Fragmentiert erhaltene Holzstelen der primären Belegungsphase TT 414 wurden im Magazin vor Ort belassen - so etwa ein Bruchstück der rechten Seite der Stele der $H r$-3s.t (Reg. $508^{43}$ ), deren Rest sich heute in London (British Museum, BM $8457^{44}$ ) befindet. Ein weiteres Stück aus der 26. Dynastie, das noch unveröffentlicht ist, kam 2007 erneut zum Vorschein: Reg. 792 aus Raum 7.1, der ursprünglichen Grabkammer des Anch-Hor (Fig. 5). Erhalten hat sich wieder nur ein Teil der rechten Hälfte inklusive des seitlichen Abschlusses mit einem bunt ausgeführten Schmuckband (erh. H. $40 \mathrm{~cm}$, B. $6,2 \mathrm{~cm}$, D. $3 \mathrm{~cm}$ ). Unter der geflügelten Sonnenscheibe im Giebel befindet sich die Darstellung eines Schreines mit Uräusfries, Hohlkehle und einer Pflanzensäule. Im Schrein steht ein Adorant nach links gewendet und darunter folgen sieben horizontale Zeilen, die jeweils nur in ihren Anfängen erhalten sind. Leider ist der Name des Besitzers verloren. Typologisch entspricht die Stele nicht den gängigen Formen, wie sie Munro für das Theben der Saitenzeit zusammengestellt hat. Reg. 792 findet aber in Boston MFA 72.4279 eine sehr nahe Parallele. Diese Stele eines WedjaHor wurde in die 26. Dynastie datiert und zeigt, bis auf die Tatsache, dass die Säulenform des Kiosks eine andere ist, genau denselben Aufbau wie das Stück aus TT $414 .^{45}$

Es scheint sich also um eine selten belegte Sonderform zu handeln. Eine weitere Entspre-

42 Bietak \& Reiser-Haslauer 1982, 183. Für einen namentlich bekannten jmj-hnt $n$ dw3.t-ntr Jrt-hr-rw, der vielleicht der Sohn (oder Schwiegersohn) des AnchHor war, s. BIETAK \& Reiser-Haslauer 1982, 251.

43 Bietak \& Reiser-Haslauer 1982, 251 und G 111.

44 Bietak \& Reiser-Haslauer 1982, Taf. 155; Bierbrier 1987, 23, Taf. 38-39.

45 S. LEPROHON 1991, 33-35. LeProHON nennt als Parallelen aus Theben Kairo A 9420 (Munro 1973, Abb. 14), Kairo A 9915 (Munro 1973, Abb. 20), Kairo A 9417 (ZAYED 1968, Taf. 16B; Munro 1973, Abb. 21; alle vom Typ Theben II A.S. aus der 26. Dyn.). Bis auf die Stele Kairo A 9417, die halbplastische Zeltstangensäulen besitzt, zeigen diese Stücke aber m.E. keine Ähnlichkeiten.

46 Munro 1973, Abb. 45; Bierbrier 1987, 30, Taf. 54-55. chung, die sich allerdings dahingehend unterscheidet, dass die architektonischen Bestandteile des Schreines nicht wie bei Reg. 792 zweidimensional und gemalt sind, sondern halbplastisch ausgearbeitet wurden, kam erstaunlicherweise ebenfalls im Grab des Anch-Hor zum Vorschein. Es handelt sich um BM 8482, ${ }^{46}$ die Stele eines Harwa, der wohl als Bruder des Anch-Hor zu identifizieren ist. ${ }^{47}$ Reg. 792 und BM 8482 zeigen exakt dieselbe Säulenform (nämlich Zeltstangensäulen), nur eben hier als gemalte Wiedergabe und dort als Halbsäule. Es ist demnach verlokkend anzunehmen, dass auch der unbekannte Besitzer von Reg. 792 zur unmittelbaren Kernfamilie des Anch-Hor gehört hat.

Holzstelen lebten auch nach der 26. Dynastie fort, ${ }^{48}$ allerdings scheint sich ihr Charakter in der 30. Dynastie und der Ptolemäerzeit etwas verändert zu haben, wie es der Befund in TT 414 impliziert. Die Stelen mit plastischen Architekturteilen wie diejenige des Harwa dürften die ersten Belege dafür sein, dass diese Objektgruppe auch in ihren hölzernen Ausführungen immer mehr zu einer Kultstelle gestaltet wurde (s. u.).

\section{Fundmaterial aus der 30. Dynastie bis in die frühptolemäische Zeit}

Mit der spätsaitischen Zeit dürfte die Nutzung von TT 414 vorerst zu einem Stillstand kommen und erste Plünderungen der Bestattungen sowie Steinraub an den Reliefs setzten ein. ${ }^{49}$ Erst im 4. Jahrhundert, mit der Periode der 30. Dynastie, wurden die spätzeitlichen Monumentalgräber im Asasif insgesamt zum Leben erweckt und auch das Grab des Anch-Hor intensiv für Bestattungen genutzt. ${ }^{50}$ Wie schon in der 26. Dynastie, so dürfte auch die zweite Blütezeit des Areals im 4. Jahr-

47 VittmannN 1978, 129; GraEFE 1978, 43-44 (Harwa als wohl identisch mit einem der beiden Brüder namens Psmtk-mn-m-W3s.t, der als schöner Name diente); s. auch LEAHY 1980, 63.

48 Für die persische Zeit s. Aston 2003, 162.

49 Bietak \& Reiser-Haslauer 1978, 141. Die kleinen Anlagen im Asasif scheinen auch während der 27. Dynastie verwendet worden zu sein, allerdings ist hier der Befund noch sehr lückenhaft und umfasst in erster Linie Keramik, s. BuDKA 2006, Bd. III, 609-611; vgl. auch ähnliche Funde in TT 194 und TT 294 (s. Aston 2003, 162).

50 Zusammenfassend zu den Bestattungen der 30. Dynastie bzw. der frühen Ptolemäerzeit in Theben s. AsTon 2003, 162-163 und STRUdwick 2003, 172-174 mit Abb. 3. 
hundert v. Chr. mit kulttopographischen Bezügen, der Tradition des Talfestes und der Nähe zu Deir el-Bahari im Zusammenhang stehen,${ }^{51}$ denn abermals fungierten die Tempelgräber als Vorzugsplätze für Familien, die beruflich mit dem Tempel des Amun von Karnak verknüpft waren.

Zur Zeit der 30. Dynastie fanden mehrere Umbauten in TT 414 statt - besonders der Lichthof musste wieder instand gesetzt werden. Stützmauern und Abmauerungen aus Lehmziegeln wurden eingezogen, teilweise wurden Sargbretter aus der 26. Dynastie in diese integriert und als Architrave für Interkolumnien verwendet. ${ }^{52}$ Diese sekundäre Anwendung, die im Verlauf der Grabung in situ beobachtet werden konnte, ${ }^{53}$ belegt beispielsweise auch Reg. 08/10, ein Haussargfragment von der Seite des gewölbten Dekkels, da sich das Stück noch in einer dicken Kruste aus Lehm und Mörtel befindet. Herabgefallene Kalksteinplatten der saitischen Verkleidung des Lichthofes wurden wieder eingesetzt, allerdings ohne Berücksichtigung des Reliefs und anschließend auch mit Kalkmörtel überzogen, sodass die ursprüngliche Dekoration nicht länger sichtbar war. ${ }^{54}$

\subsection{Särge}

Im Grab des Anch-Hor gelang die bislang einzige Entdeckung einer ungestörten Bestattung aus dem 4. Jahrhundert v. Chr. in Theben: diejenige des Wach-jb-Ra $\left(W 3 h-j b-R^{\subsetneq}\right) .^{55}$ Für Wach-jb-Ra ist ein bereits bestehender Schacht erweitert worden und eine neue Kammer an dessen Sohle ausgehauen worden (Raum 10.2). Andere Funde zeigen aber auch, dass bereits bestehende Kammersysteme wiederbenutzt wurden, so v.a. die Bestattungsanlage des Anch-Hor selbst (Raum 7.1). Von dort stammen polierte Naturholzsärge mit geschnitzter Dekoration sowie bunt bemalte

${ }^{51}$ Zur Bedeutung des Talfestes für die Entwicklungsgeschichte des Asasif s. BIETAK, in: BIETAK \& REISER-HasLAUER 1978, 19-29.

${ }^{52}$ Für eine detaillierte Beschreibung dieser Bauphase im Lichthof s. BietaK \& Reiser-HASLAuer 1978, 141-146.

53 S. Bietak \& Reiser-Haslauer 1978, Taf. 27; vgl. auch Abb. 77.

54 Diese Behandlung der Bausubstanz und der Funde aus der primären Phase steht etwas im Gegensatz zu Textbelegen aus dem Raum Memphis, wo sich Wiederbenutzer damit rühmen, die Anlagen wiederherzustellen und den Namen des ursprünglichen Besitzers dabei „dauern zu lassen“; s. JANSEN-WINKELN 1997. anthropoide Särge, die in der 30. Dynastie und der frühptolemäischen Zeit angefertigt wurden. ${ }^{56}$ Die Holzsärge, welche naturbelassen blieben und einen Blütenkragen und Inschriftkolumnen in geschnitzter Technik aufweisen, waren mehrheitlich aus Tamariskenholz und seltener aus einer noch nicht sicher identifizierten Art von Nadelholz hergestellt. ${ }^{57}$ Die aktuellen Arbeiten im Magazin zeigten, dass es sich bei diesem Typ von Särgen nicht nur um innere anthropoide Särge handelt ${ }^{58}$ sondern dass auch äußere Särge und Haussärge in dieser Technik gestaltet wurden. So muss beispielsweise Reg. 07/40, die Stirnseite eines Sarges aus Tamariskenholz, aufgrund seiner beachtlichen Maße von $57 \times 20,5 \mathrm{~cm}$ und der groBen Brettstärke von $7 \mathrm{~cm}$ zu einem äußeren anthropoiden Sarg gehört haben.

Im Inneren von Reg. 614, einem in der Grabkammer des Anch-Hor bestatteten anthropoiden Innensarg aus poliertem Tamariskenholz, gelang während der Restaurierungsarbeiten eine bemerkenswerte Entdeckung. Die Bestattung war zwar geplündert, die Mumie geraubt worden, aber Reste des Oberkörpers hatten sich noch auf der mit Bitumen überzogenen Bodenplatte erhalten. Auf der linken Seite der Mumienreste kamen der vollständige Abdruck und Bruchstücke mehrerer Lagen einer Papyrusrolle zum Vorschein, wobei neben hieratischen Textresten auch eine bunt ausgeführte Vignette, die nach links schreitende männliche Gottheiten zeigt, sichtbar ist. Dass die Mitgabe von Totenbuchpapyri zu dieser Zeit Standard war, belegen einerseits der ungestörte Befund des Wach-jb-Ra, ${ }^{59}$ andererseits die oben zitierte Beschreibung von Lepsius (,fast jede Mumie hatte einen dicken (Toten-)Papyrus neben sich"). Die Positionierung der Papyrusrollen kann dabei alternieren: neben der Mumie wie bei Reg. 614 und laut Lepsius oder auf der Mumie, in

55 Ausführlich zu diesem Befund s. BIETaK \& Reiser-HasLAUER 1982, 199-220 sowie zusammenfassend bei GRAJETZKI 2003, 119-121.

56 Bietak \& Reiser-Haslauer 1982, 166.

${ }^{57}$ Die Identifizierung der Holzarten verdanke ich Erico Peintner.

58 So Bietak \& Reiser-Haslauer 1982, 166, da es einige Belege dafür gibt, dass Personen desselben Namens einen äußeren bunt bemalten und einen inneren naturbelassenen Sarg besitzen.

59 BietaK \& Reiser-Haslauer 1982, 212-214, Abb. 97. 


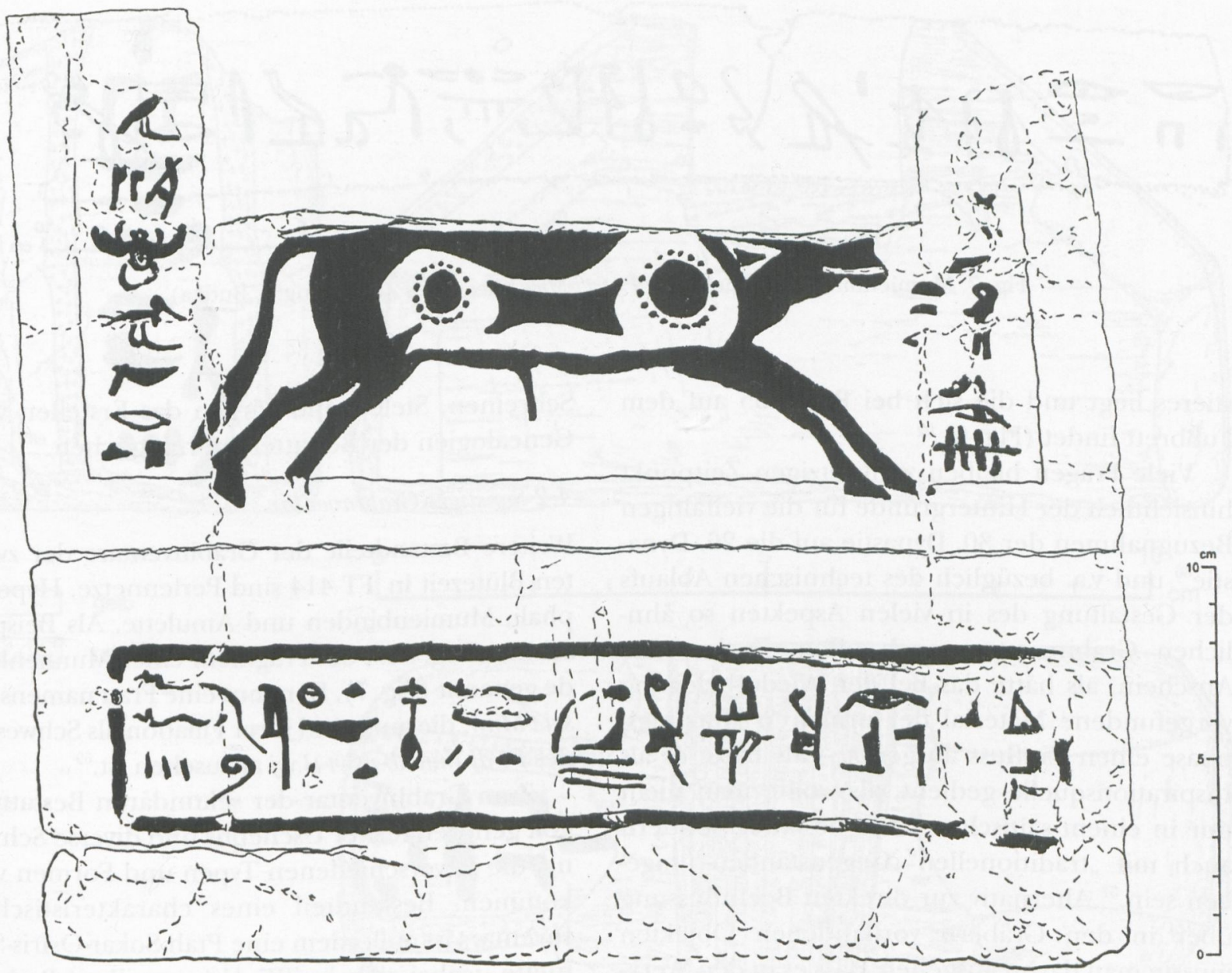

Fig. 6 Das Fußbrett des Sarges Reg. 655 (1:2, Zeichnung: S. Bock)

deren Wickel eingebunden (so bei Wach-jb-Ra). ${ }^{60}$ Aus welchen Gründen eine bestimmte Position den Vorzug bekam, bleibt unklar - der nebeneinander auftretende Befund in TT 414 zeigt, dass es weder auf einer lokalen Tradition beruht noch ein chronologisches Indiz ist.

Der schön bemalte innere Sarg des Amunprie-

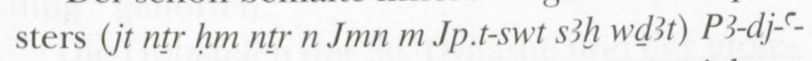
$s$ (Reg. $655^{61}$ ) wurde 2007 restauriert, gezeichnet und gemeinsam mit seinem äußeren, schwarz bemalten Sarg ins Magazin der Antikenverwaltung überführt. ${ }^{62}$ Der innere Sarg gehört zu einer Gruppe von mindestens elf bunt bemalten

${ }^{60} \mathrm{Zu}$ diesem Befund s. zuletzt Martin \& RyHOLT 2006, 273-274.

${ }^{61} \mathrm{Zu}$ den vollständigen Titeln und der Genealogie s. Reiser-Haslauer, in: Bietak \& Reiser-Haslauer 1982, 255 und 276 (G 79).

${ }^{62}$ Zur Fundposition dieser beiden Särge in der Grab- anthropoiden Holzsärgen aus der 30. Dynastie bis in frühptolemäische Zeit, die in der ursprünglichen Grabkammer des Anch-Hor bestattet wurden. ${ }^{63}$ Die Besitzer gehörten zu einer weitverzweigten Familie von Amunpriestern rund um $P_{3}$ dj-jmn-nb-nswt-t3.wj. ${ }^{64}$ Das Stück griff viele Motive und Bildinhalte aus der Spätzeit auf und illustriert so anschaulich den „Archaismus", der für die thebanische Sargentwicklung dieser Epoche charakteristisch, aber noch nicht in vollem Umfang verstanden ist. Das vielleicht beste Beispiel für die Übernahme alter Bildinhalte ist die Szene der Mumie, die auf dem Rücken des Apis-

kammer des Anch-Hor (Raum 7.1) s. BIETAK \& REISERHaslauer 1982, 165, Abb. 67 und 68.

63 Bietak \& Reiser-Haslauer 1982, 166.

${ }^{64}$ S. die rekonstruierten Genealogien bei MunRo 1973, 57-58; Bietak \& Reiser-Haslauer 1982, 252-256. 


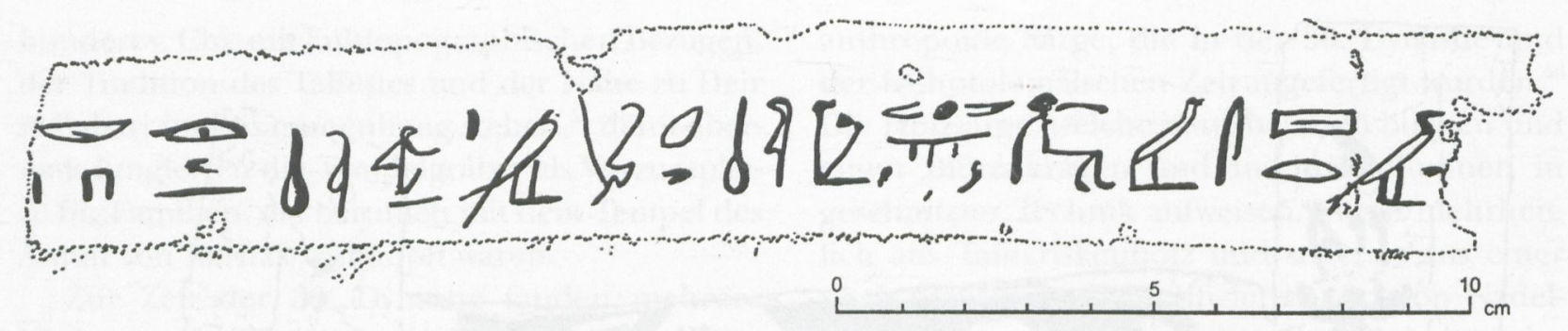

Fig. 7 Mumienbindenfragment der T3-šr.t-Mnw (Reg. 545, Zeichnung: J. Budka)

stieres liegt und die sich bei Reg. 655 auf dem Fußbrett findet (Fig. 6). ${ }^{65}$

Viele Fragen bleiben zum jetzigen Zeitpunkt hinsichtlich der Hintergründe für die vielfältigen Bezugnahmen der 30. Dynastie auf die 26. Dynastie $^{66}$ und v.a. bezüglich des technischen Ablaufs der Gestaltung des in vielen Aspekten so ähnlichen Grabinventars noch offen. Es hat den Anschein, als hätte das bei der Wiederbelegung vorgefundene Material der primären Nutzungsphase einen Einfluss ausgeübt - als hätte es als Inspirationsquelle gedient, als wollte man nicht nur in einem saitischen Grab bestattet, sondern auch mit "traditionellen" Gegenständen umgeben sein. ${ }^{67}$ Alternativ zur direkten Beeinflussung über in den Gräbern vorhandenen Objekten müsste man davon ausgehen, dass es in den Werkstätten noch Vorlagen und Musterbücher gegeben hat, welche die mindestens 150 Jahre alten Traditionen und Formen beschrieben.

Klärungsansätze könnte hier eine detaillierte Analyse des Materials aus dem Grab des AnchHor unter Berücksichtigung der Neufunde im Magazin liefern, da in TT 414 nicht nur das einzige ungestörte Begräbnis der 30. Dynastie in Theben entdeckt wurde $\left(W 3 h-j b-R^{\top}\right)$, sondern zudem Inschriften auf zahlreichen Opferplatten,

65 Diese Gestaltung des Fußbrettes ist seit der 22. Dynastie in Theben belegt und war besonders in der 25 . und der 26. Dynastie beliebt; s. TAYLOR 1989, 61 .

66 Zur starken Anlehnung der 30. Dynastie an die 26. Dynastie, die in vielen Bereichen greifbar ist, s. JANSENWinKELN 1997, 176 mit weiterer Literatur in Anm. 15.

67 Es gibt auch Indizien, die dafür sprechen, dass saitische Särge in den späten Nutzungsphasen wiederverwendet wurden, s. BietaK \& Reiser-Haslauer 1982, 154-155.

68 S. bereits das von REISER-HASLAUER zusammengestellte genealogische Register, in: BIETAK \& REISER-HASLAUER 1982, 267-282 (für erweiternde Literatur zu den intrusiven Bestattungen s. FN 12).

69 Munro 1973, 57 (Mumienbinden Louvre X 24 und
Schreinen, Stelen und Särgen das Erstellen von Genealogien der Bestatteten ermöglichen. ${ }^{68}$

\subsection{Sonstiges Grabinventar}

Weitere Bestandteile der Grabinventare der zweiten Blütezeit in TT 414 sind Perlennetze, Hypocephali, Mumienbinden und Amulette. Als Beispiel sei hier Reg. 545, das Fragment einer Mumienbinde genannt (Fig. 7). Es nennt eine Frau namens T3srj.t-Mnw, die aufgrund ihrer Filiation als Schwester des P3-dj-Jmn-nb-nswt-t3.wj anzusehen ist. ${ }^{69}$

Zum Grabinventar der sekundären Bestattungen gehören ferner Uschebtis und diverse Schreine, die in verschiedenen Typen und Formen vorkommen. Bestandteil eines charakteristischen Inventars ist außerdem eine Ptah-Sokar-Osiris-Statuette, wobei sich in TT 414 vor allem Podeste derartiger Statuetten erhalten haben. ${ }^{70}$ Bis auf die neu entwickelten Hypocephali sind dies also alles Gegenstände, die bereits zu saitischen Bestattungen gehörten und in dieser Tradition fortlebten, wenn sie auch stilistisch und in ihrer Formgebung modifiziert wurden. ${ }^{71}$

\subsection{Holzstelen}

Ein weiteres Beispiel für die Umgestaltung und Neuadaptierung traditioneller Grabbeigaben

25); Bietak \& Reiser-Haslauer 1982, G 147; Bierbrier $1987,37$.

70 Vgl. die oben genannte Bemerkung von LEPSIUS; viele dieser Statuetten dürften also frühen Antikensammlern und der Suche nach Papyri zum Opfer gefallen sein.Als Beispiel kann eine Ptah-Sokar-Osiris-Statuette einer 3s.t$m$-3h-bj.t genannt werden, die aus TT 414 stammt und sich als Dauerleihgabe des Louvre (N 670) heute im Nationalmuseum in Warschau befindet (inv.nr. 143346), s. LIPINSKa 2008. Das zu dieser Statuette gehörige Podest wurde bei den österreichischen Grabungen gefunden und 2007 im Magazin lokalisiert (Reg. 695).

71 Zur Charakterisierung des Inventars im 4. Jh. v. Chr. s. Aston 2003, 162-163. 

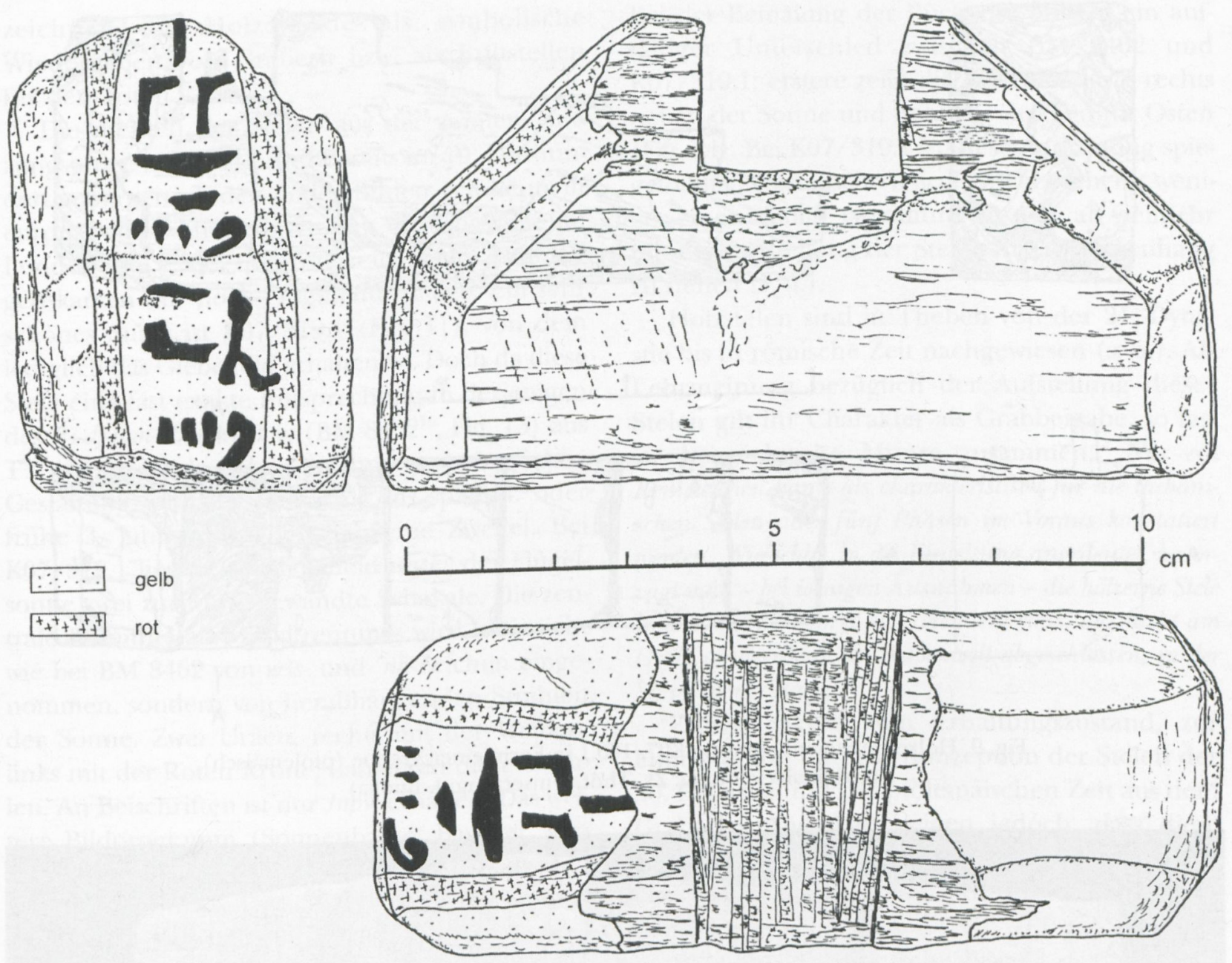

Fig. 8 Hölzerne Stelenhalterung K07/188.1 mit Beschriftung (30. Dyn. bis frühptolemäisch) (Originalzeichnung: A. Korhonen, Umsetzung: J. Budka)

sind Holzstelen, deren Entwicklung in TT 414 gut nachvollzogen werden kann. Sie sollen im Folgenden abgesetzt von den übrigen Beigaben des 4. und frühen 3. Jahrhunderts vorgestellt werden, da sie funktional nun wohl eher zur Grabausstattung ${ }^{72}$ gehörten.

Die Holzstelen der 30. Dynastie und der ptolemäischen Zeit aus dem Grab des Anch-Hor nahmen mit einer Höhe von über $50 \mathrm{~cm}$ wieder gröBere Dimensionen als die der 26. Dynastie oder der Dritten Zwischenzeit an. Ihre Formgebung ändert sich ebenfalls signifikant: seit der 30 .

$\overline{72}$ Zur Unterscheidung von Grabbeigaben als bewegliche Gegenstände und Grabausstattung als unbewegliches Inventar s. AltenMüLLER 1977, 837.

73 S. BiERBriER 1987, Taf. 74-75. Eine ähnliche Halterung befindet sich heute in Cambridge, s. MARTIN 2005, 125,
Dynastie sind hölzerne Halterungen belegt, die zum Aufstellen der Stele dienten. Eine offenbar frühe Form stellen halbrunde Halterungen dar, die auf der Frontseite beschriftet sind (Fig. 8). Das hier gezeigte Stück K07/188.1 aus dem Füllschutt von TT 414 findet die besten Entsprechungen in den Halterungen der Stele des Wachjb-Ra (BM 8461, s.u.). ${ }^{73}$ Es ist über einer weißgelblichen Grundierung teilweise gelb und rot bemalt. Die Beschriftung, die wohl den Ausschnitt einer Opferformel darstellt, wurde in schwarz ausgeführt.

Nr. 85 (Acc. No. E. 21.1919); dieses Stück (dessen Kenntnis ich dem freundlichen Hinweis von Marc LOTH verdanke) wurde in die Dritte Zwischenzeit datiert, was angesichts der Belege aus sicherem Kontext in TT 414 zu früh wirkt. 

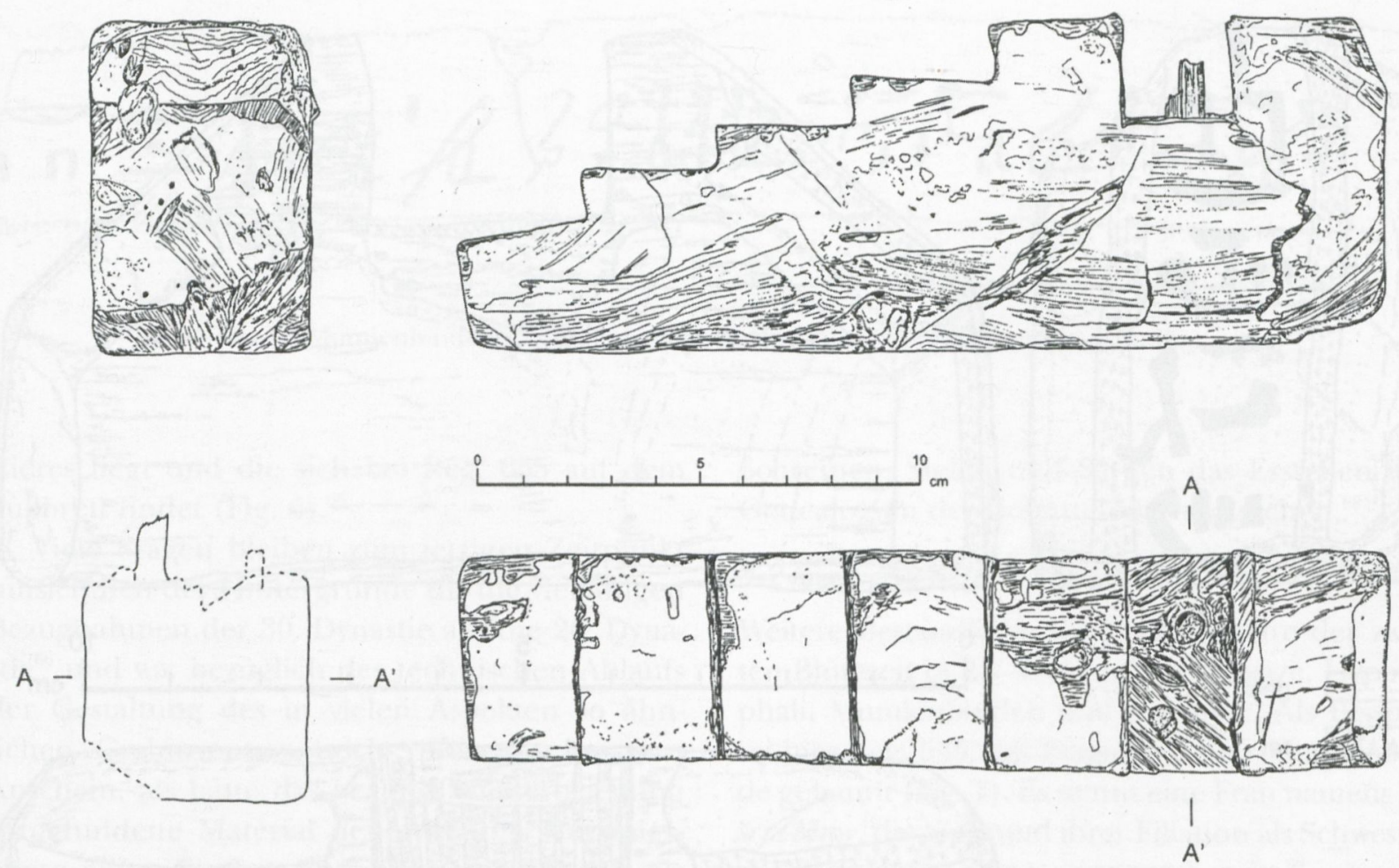

Fig. 9 Hölzerne Stelenhalterung K07/180.1 in Form einer Treppe (ptolemäisch)

(Originalzeichnung: Zs. Thót, Umsetzung: J. Budka)

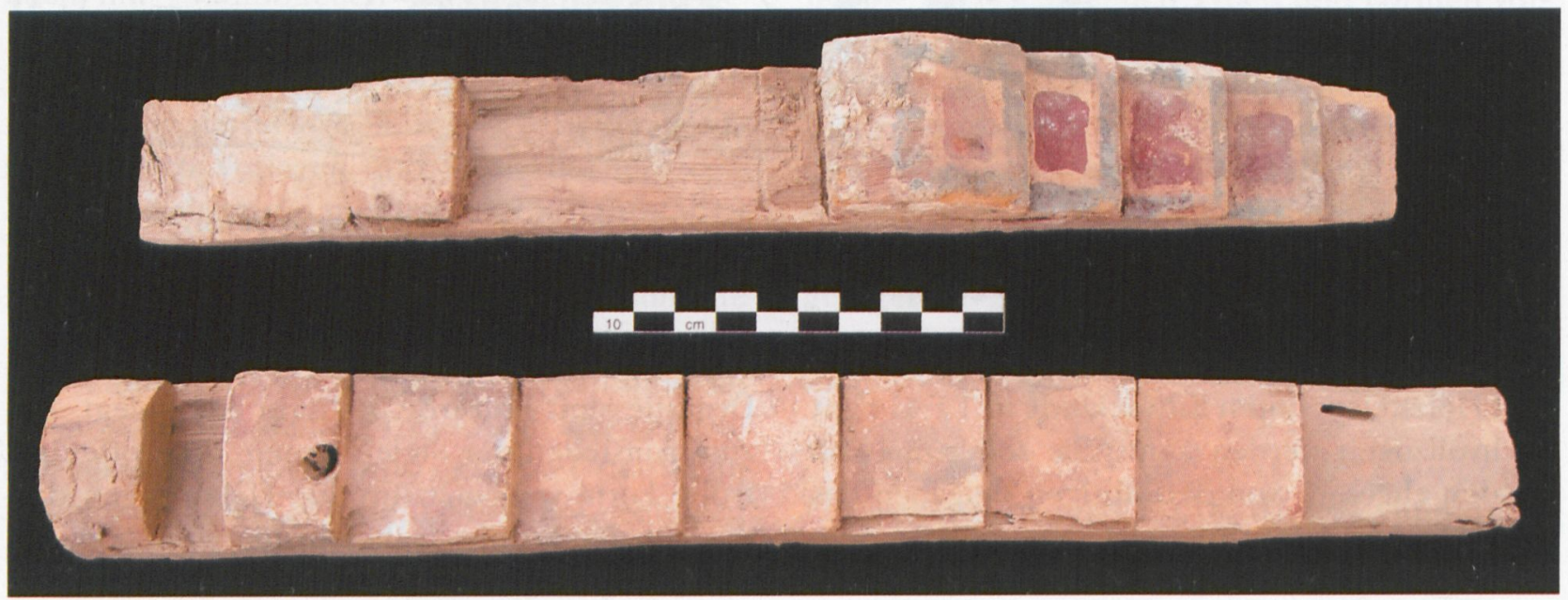

Fig. 10 Hölzerne Stelenhalterungen in Form von Treppen (K07/302.2 und K07/302.3)

Die Stelenfüße, die im weiteren Verlauf meist treppenförmig (Figs. 9-10) gestaltet sind, gehörten in der Ptolemäerzeit zum Standard einer Holzstele - ebenso wie die kleine, rundplastische Holzstatuette eines Bavogels, die auf dem Stelengiebel angebracht war. ${ }^{74}$ Gleichzeitig mit dem Aufkommen der Stelenfüße wurde jetzt auch häufiger die Rückseite dekoriert (meist mit der
Sonnenscheibe und dem Zeichen für Osten und Westen).

Die Konzeption der Stelen als aufrecht (und frei) stehende Denkmäler mit „Treppenaufgang“, Bavogel und einem teils mit Särgen vergleichbaren Bildprogramm (z.B. die Dekoration des Giebelfeldes, Sonnenbarke, Anubis-Schakale, Götterreihen, Bavögel als Adoranten etc.) kenn-

${ }^{74}$ MunRo 1973, 177-178. 
zeichnen die Holzobjekte als symbolische Wiedergaben von Gräbern bzw. als Kultstellen für den Toten. ${ }^{75}$

Die meisten der Stelen aus der zweiten Nutzungsphase in TT 414 sind bereits im 19. Jahrhundert geborgen worden und befinden sich heute in europäischen Museen, v.a. im British Museum London (s.o.). Bei den österreichischen Grabungen kamen nur noch Fragmente zutage. Ein sehr schönes Stück ist K07/319.1 (Fig. 11), von dem lediglich das Giebelfeld erhalten ist. Doch da diese Stele eine fast exakte Entsprechung in derjenigen des P3-dj-jmn-nb-nswt-t3.wj (BM 8462 ${ }^{76}$, Fig. 13) aus TT 414 findet, bestehen an ihrer ursprünglichen Gestaltung und der Datierung ins späte 4. oder frühe 3. Jahrhundert v. Chr. keine Zweifel. Bei K07/319.1 liegen im Giebelfeld unter der Flügelsonne zwei zur Mitte gewandte Schakale. Die zentrale Kolumne als Mitteltrennung wird hier nicht wie bei BM 8462 von $w 3 s$ - und ${ }^{n h}$-Zeichen eingenommen, sondern von herabhängenden Strahlen der Sonne. Zwei Uräen, rechts mit der Weißen, links mit der Roten Krone, flankieren diese Strahlen. An Beischriften ist nur Jnpw erhalten. Das weitere Bildprogramm (Sonnenbarke, Bavögel, Verstorbener vor Götterreihe) sowie die Textzeilen können nach dem Vorbild von BM 8462 ergänzt werden. Die Identität des Besitzers muss offen bleiben, doch es wird sich wohl um ein Familienmitglied des P3-dj-jmn-nb-nswt-t3.wj gehandelt haben. Wie BM 8462, so hat auch K07/319.1 ehemals auf zwei Stelenfüßen gestanden. Solche Füße wie sie bei der Stele des P3-dj-jmn-nb-nswt-t3.wj noch im Verband erhalten sind - als Doppeltreppe mit jeweils vier Stufen - sind zweifach im Magazinbestand aus TT 414 belegt (K07/149.5 und K07/302.2, Fig. 10). Diese Halterungen, die auf beiden Seiten eine Treppe aufweisen sowie die schön bemalten Rückseiten der Stelen sprechen dafür, dass die Objekte frei im Raum aufgestellt wurden und beide Dekorflächen sichtbar waren.

75 So auch TAYLOR 2001a, 163, zu Abb. 118: „The ba of the deceased is perched on the top vault, stressing the role of the stela as a miniature representation of the tomb." Möglicherweise stehen die Schließsteine der Lokulusgräber der römischen Zeit (vgl. CoRBELli 2006, 33-34 mit Abb. 34) an einem Endpunkt dieser Entwicklung.

76 Bierbrier 1987, 36-38.

77 Munro 1973, 10. Ähnlich zum grundsätzlichen Beigabencharakter der Holzstelen zuletzt auch MiNAS-NERPEL 2006, 330.
Bei der Bemalung der Rückseite besteht ein auffälliger Unterschied zwischen BM 8462 und K07/319.1: erstere zeigt die West-Standarte rechts neben der Sonne und links das Zeichen für Osten (Fig. 14). Bei K07/319.1 ist diese Anordnung spiegelbildlich vertauscht (Fig. 12). Dies scheint weniger eine zufällige Gestaltung zu sein, als vielmehr mit der Aufstellung der Stelen in Zusammenhang zu stehen (s. u.).

Holzstelen sind in Theben von der 21. Dynastie bis in römische Zeit nachgewiesen (s. o.). Als Lehrmeinung bezüglich der Aufstellung dieser Stelen gilt ihr Charakter als Grabbeigabe, so fasste beispielsweise Munro zusammen: „Nur ein Kennzeichen kann als charakteristisch für die thebanischen Stelen aller fünf Phasen im Voraus konstatiert werden. Wie schon in der Einleitung angedeutet, bevorzugt man - bei wenigen Ausnahmen - die hölzerne Stele und damit jene Form des Totendenkmals, das nicht am Grab, sondern von der Außenwelt abgeschlossen, in der Sargkammer stand." ${ }^{\text {T7 }}$

Einige Fakten zum Erhaltungszustand, zur Fundposition und zur Konzeption der Stelen der 30. Dynastie und der ptolemäischen Zeit aus dem Grab des Anch-Hor zeigen jedoch, dass diese Annahme wohl zu überdenken ist.

1. Im österreichischen Konzessionsgebiet wurden Holzstelen primär in TT 414 gefunden; in den kleinen Anlagen fehlt bislang der Nachweis für diese Objektgruppe. ${ }^{78}$ Dies könnte ein Indikator für unterschiedliche soziale Gruppen sein, es könnte aber auch mit der Funktion bzw. der Aufstellung(smöglichkeit) der Objekte zusammenhängen.

2. Bei den österreichischen Grabungen wurden ausschließlich Stelen aus der 26. Dynastie in den Schachtfüllungen gefunden. Die wenigen Fragmente späterer Holzstelen - die häufigsten Belege liegen in der Form von Stelenfüßen vor (Figs. 8-10) - wurden hingegen im allgemeinen Füllschutt der unterirdischen Kultanlage geborgen. ${ }^{79}$

\footnotetext{
78 Allerdings fand sich in Grab VII eine Art Stelenhalterung aus Holz (K 124).

79 Grundsätzlich wurde die gesamte Füllung des Grabes mehrfach umgelagert und vermischt, weshalb die Fundposition von Gegenständen nur unter Vorbehalt für Rekonstruktionen herangezogen werden kann; s. BietaK \& Reiser-Haslauer 1978, 75.
} 


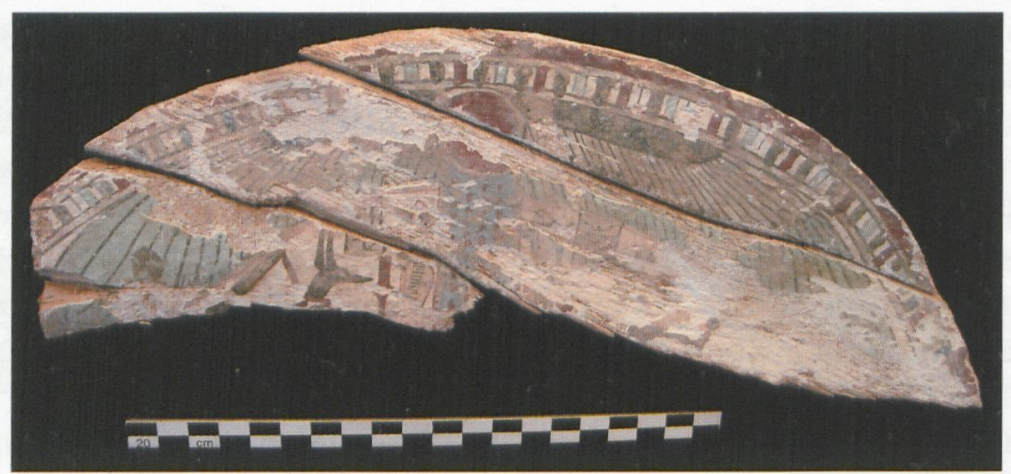

Fig. 11 Das Stelenoberteil K07/319.1

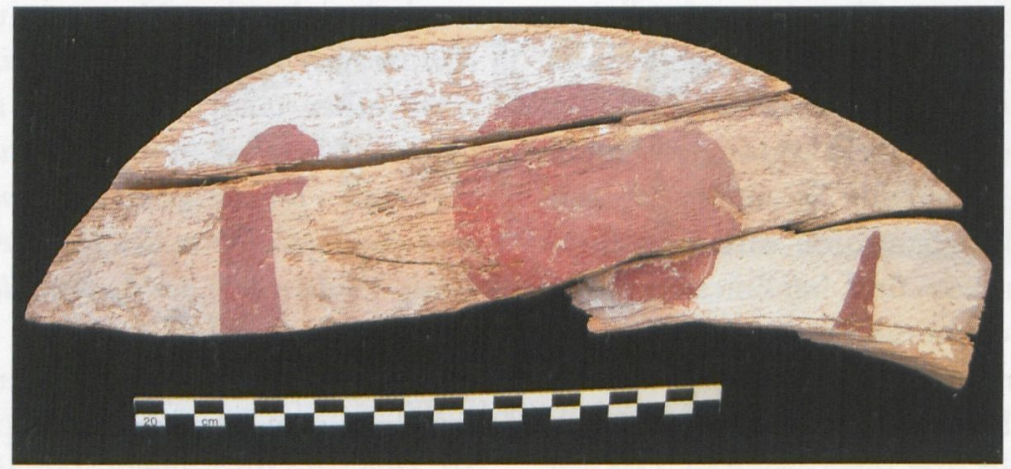

Fig. 12 Die Rückseite des Stelenfragments K07/319.1

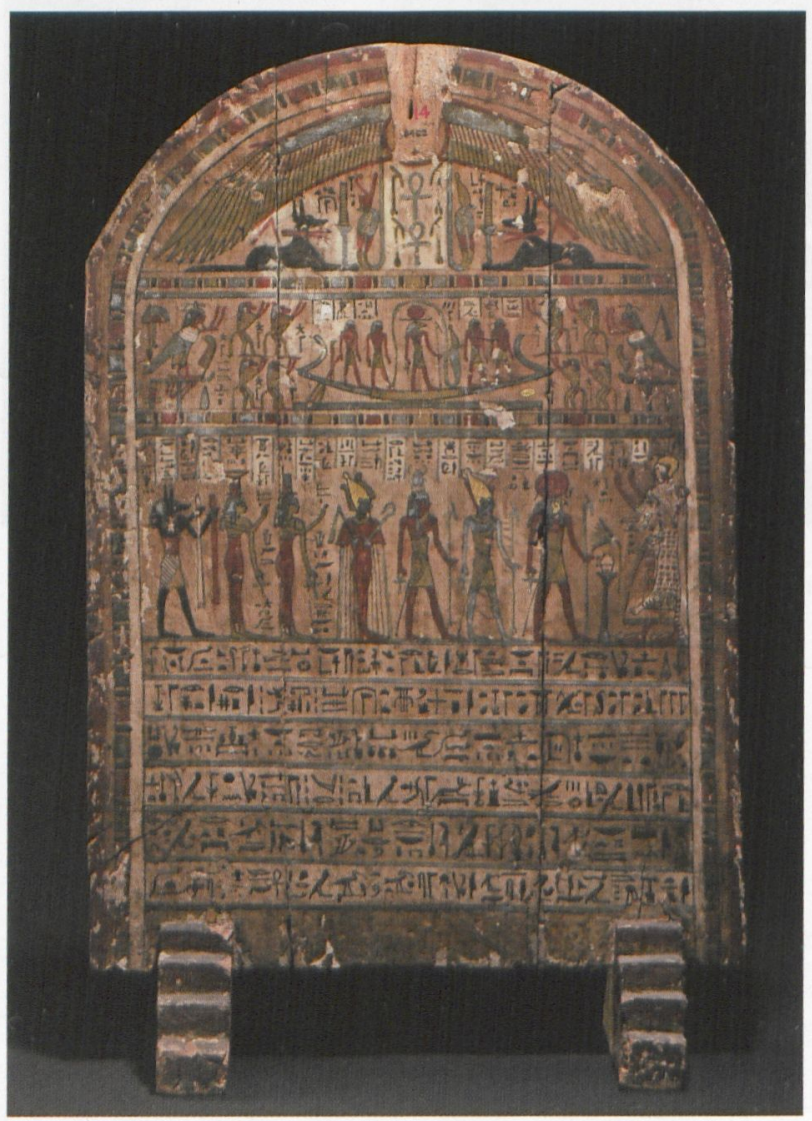

Fig. 13 Die Stele des P3dj-jmn-nb-nswt-t3.wj BM 8462, (C) British Museum London

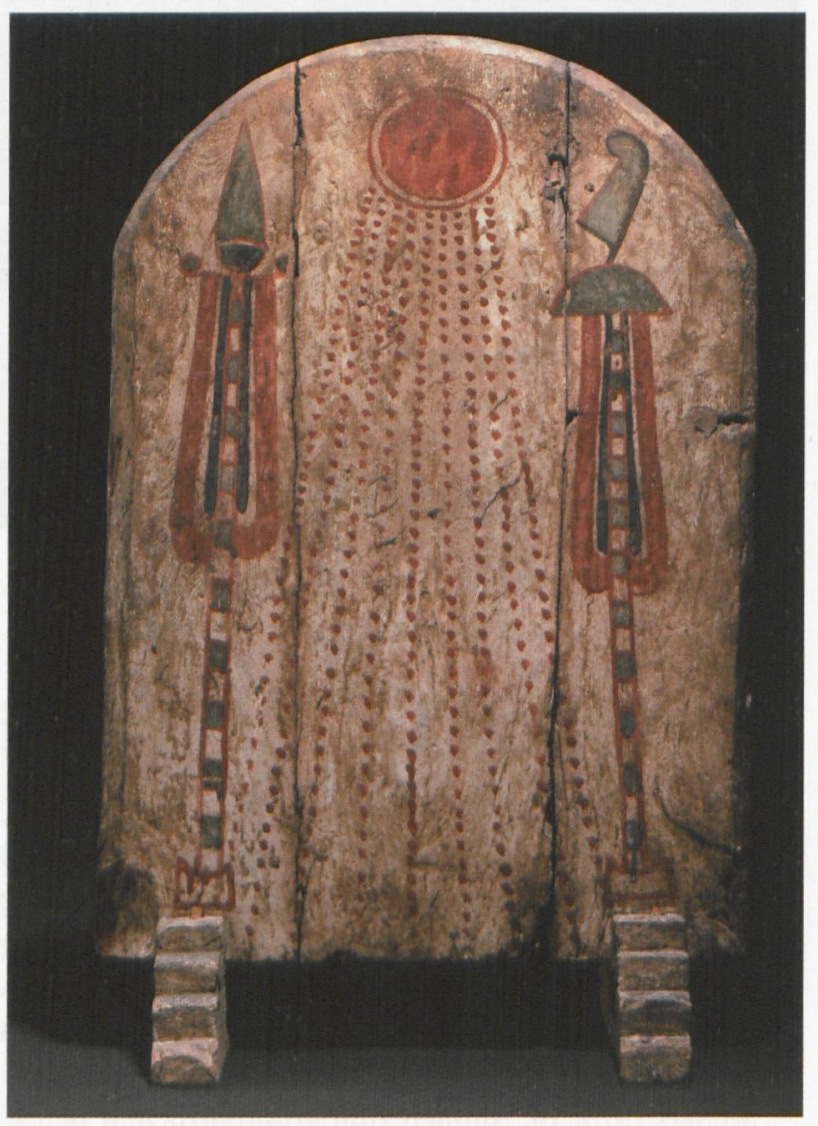

Fig. 14 Die Rückseite der Stele BM 8462, (C) British Museum London 
3. Eine große Gruppe von späten Holzstelen wurde bereits im 19. Jahrhundert in TT 414 gefunden (deutlich mehr als aus der 26. Dynastie).$^{80}$ Diese sind meist intakt, inklusive ihrer Füße. Wie oben dargelegt wurde, wurden bei diesem frühen Aufsammeln von Funden die Bestattungsanlagen des Grabes nicht erreicht, hingegen konzentrierte man sich auf den Lichthof, die Räume im vorderen Abschnitt und später auch auf den Pfeilersaal (Raum 4). Am deutlichsten wird dies bei der intakten Stele des Wach-jb-Ra (BM 8461 ${ }^{81}$ ): beinahe unversehrt 1823 in London angekauft, dauerte es bis in die 1970er, dass die Bestattung des Wach-jb-Ra ihrerseits in ungestörtem Zustand freigelegt werden konnte. ${ }^{82}$

4. Die Gestaltung der Holzstelen durch Treppenpodeste zu Opferkultstätten findet Vorläufer in der 25. und 26. Dynastie: in den Lichthöfen mehrerer Monumentalgräber standen steinerne Altäre mit einem treppenförmigen Podest, bekrönt von einer Stele mit Sonnenhymnus (so bei Harwa und Scheschonk sowie im zweiten Hof des Oberbaus bei Ibi nachweisbar). ${ }^{83}$

Diese vier Aspekte sprechen dafür, dass der Aufstellort der späten Stelen aus Holz nicht mit dem Abstellplatz der Särge identisch war. Da derartige Stelen mit Füßen und Bavögeln nur aus monumentalen Tempelgräbern belegt sind, scheint ihre Aufstellung entweder eine spezifische Raumeinheit erfordert zu haben, die in nichtmonumentalen Anlagen fehlte, oder einfach mit den jeweiligen Platzverhältnissen in Verbindung zu stehen. Dass Belzoni die große Gruppe von Stelen bergen konnte, ohne in die Tiefen des Grabes vorzudringen, ließe sich sehr gut mit einer Fundposition im Bereich des Lichthofes oder des vorderen Abschnittes der unterirdischen Anlage vereinbaren. Für die Räume 1 und 2, in denen übrigens noch während der österreichischen Grabung Relikte von Stelen in der Gestalt kleiner Bavögel zutage kamen, ${ }^{84}$ würde als Aufstellort sprechen, dass es dort Kultstellen in Form von Nischen/

\footnotetext{
80 Bierbrier 1987, 36-39.

81 Bierbrier 1987, 38-39.

82 Die Grabkammer (Raum 10.2) wurde noch mit intakter Torvermauerung angetroffen (BIETAK \& REISERHaslauer 1982, 183) - so ist es definitiv auszuschlieBen, dass die Stele BM 8461 einst in der Grabkammer stand.

83 Zu diesen Kultstellen s. EIGNER 1984, 120 und 191-192. Zum Befund bei Ibi s. GraEFE 1990, 20 und Abb. 19.
}

Scheintüren gab und es abgeschlossene Felsräume ohne Lichteinfall sind. Der Lichthof war aber im 4. Jahrhundert von Lehmziegelmauern unterteilt worden, sodass die empfindlichen Holzstelen auch dort nicht notwendigerweise dem Sonnenlicht ausgesetzt gewesen wären. Mit dem vielschichtigen Symbolismus der Stelen, der durch die Konzeption mit Bavogel und die komplexe Dekoration sowie Texte wie Opferformeln aber auch Sonnenhymnen $^{85}$ deutlich wird, ließe sich der Lichthof, der als Re-Osiris-Kultstätte zu interpretieren ist und in dem wichtige Kulthandlungen vollzogen wurden ${ }^{86}$ jedenfalls gut vereinbaren. Bereits die Stelen der Dritten Zwischenzeit brachten solar-osirianische Konzepte zum Ausdruck, dies wurde im Verlauf der Spätzeit sogar gesteigert. ${ }^{87}$ So lassen sich ja auch steinerne Vorbilder für die Stelen mit Treppenpodesten in den thebanischen Monumentalgräbern greifen, die im Lichthof aufgestellt waren. Der Lichthof blieb auch während der 30. Dynastie der Aufstellort von Opferplatten - die Holzstelen wären ergänzend dazu als individuelle Kultstellen für die bestatteten Personen zu interpretieren. Aus nicht-monumentalen Spätzeitgräbern im Asasif fehlt bislang der Nachweis von Holzstelen - diese Bauten besitzen auch weder einen Lichthof noch weitläufige unterirdische Kultanlagen oder Kultstellen.

Zusammenfassend kann vermutet werden, dass die Holzstelen der 30. Dynastie und der Ptolemäerzeit in TT 414 nicht im Bestattungstrakt, sondern im zugänglichen Bereich der unterirdischen Anlage, primär im Lichthof, sowie auch im vorderen Bereich der Felsräume (Räume 1 und $2^{88}$ ), aufgestellt waren. Die dekorierten Rückseiten wie bei BM 8462 und K07/319.1 (Figs. 12, 14) sprechen dafür, dass die Himmelsrichtungen dabei berücksichtigt wurden. Demgemäß wäre BM 8462 ehemals im nördlichen Bereich des Lichthofes (oder von Raum 2) mit Blickrichtung nach innen (zu den Kulthandlungen) aufgestellt gewesen. K07/319.1 wäre hingegen spiegelbildlich im

\footnotetext{
84 Bietak \& Reiser-Haslauer 1978, 78 (vier Stück aus Raum 1, zwei am Durchgang zu Raum 2).

85 S. beispielsweise Millet 2004.

86 Eigner 1984, 120.

87 Vgl. MinAs-Nerpel 2006, 333.

88 In diesen an den Abgang anschließenden Räumen sind in mehreren Monumentalgräbern verschiedene Kultstellen für Gottheiten, König und Gottesgemahlin belegt, s. EIGNER 1984, 193 und Abb. 154.
} 
Süden, ebenfalls mit der Ansichtsseite nach innen zur Mitte des Hofes/Raumes abgestellt worden. Durch Besucherinschriften wissen wir, dass der Lichthof, und somit auch die ihm vorgelagerten Räume zugänglich waren. ${ }^{89}$ So haben die Holzstelen des 4. und 3. Jahrhunderts v. Chr. wieder ihre Rolle als Kommunikationsmedium erreicht - vielleicht in Zusammenhang mit der Tatsache, dass keine eigenständigen Grabbauten errichtet wurden und mit Sicherheit auf steinerne Vorbilder aus der 25. und 26. Dynastie zurückgreifend.

\section{Fundmaterial aus der ptolemäischen Zeit}

Das oben besprochene Material der Bestattungen der Familie des P3-dj-jmn-nb-nswt-t3.wj reicht bis in die frühptolemäische Zeit hinein. Auch danach kam es in TT 414 wiederholt zu Bestattungen, was sich anhand von weiteren Särgen, Schreinen, Statuetten und anderen Grabbeigaben zeigen lässt.

In diesem Abschnitt möchte ich kurz auf die Fundgruppe der Keramik eingehen - der Großteil des keramischen Materials aus TT 414 datiert nämlich bereits aus der ptolemäischen Epoche. Die Auswertungsmöglichkeiten von Keramik sind bekanntermaßen sehr vielseitig. Sie reichen von Datierungshinweisen bis zu sozialen Aspekten und vor allem funktionalem Gebrauch von Strukturen.$^{90}$ Letzteres scheint hier erwähnenswert - wir können die keramischen Hinterlassenschaften in TT 414 dafür nützen, um Fragen des Totenkults, osirianische Aspekte und das wichtigste Festereignis der Nekropole, das Talfest, anzuschneiden.

89 S. Kuhlmann 1973; Eigner 1984, 118.

90 Vgl. Orton \& Tyers \& Vince 1993, 23.

91 S. etwa den Papyrus des Nesmin, pBM 10209 (HaIkAL 1970); eventuell sind in ptolemäischer Zeit schon einige Handlungen im Vergleich zum Neuen Reich und der Spätzeit modifiziert worden, weshalb späte Quellen nur mit Vorbehalt für Rekonstruktionen in älteren Epochen herangezogen werden dürfen, vgl. WIEBACH 1986, 265. So scheint es auch ab der Spätzeit zu einer Art Fusion mit dem Dekadenfest gekommen zu sein, s. Traunecker \& le Saout \& Masson 1981, 134-137.

92 Vgl. Batallle 1951, 337.

93 Vgl. Bataille 1951, 333; Bataille 1952, 269-270; HaIKal 1970, Bd. II, 15 und Graefe 1986, 188.

94 Haikal 1970, Bd. II, 15 und Graefe 1986, 188 mit Anm. 18.

95 So bereits Graefe 1986, 188.
Das Talfest hat seine wichtige Rolle als thebanisches Totenfest bis in ptolemäische Zeit beibehalten. ${ }^{91}$ Davon zeugen zahlreiche textliche Quellen. ${ }^{92}$ Es war zu dieser Zeit unter dem Namen „Die Überfahrt des großen Gottes Amun nach Memnonia“" bekannt. ${ }^{93}$ Mehrere Papyri aus römischer Zeit nennen das Talfest und die zu diesem Anlass zu zitierenden Sprüche, ${ }^{94}$ weshalb die Festtradition offenbar mit der Integration Ägyptens ins Römische Weltreich kein Ende fand. Allerdings wird Amun nicht mehr ausdrücklich erwähnt, so scheint der Osirisglaube, der im Verlauf des 1. Jahrtausends v. Chr. zunehmend wichtiger wurde, hier deutlich eingedrungen zu sein..$^{95}$

In diesem Zusammenhang sind hunderte kleiner Räucherschalen und Kelche aus TT 414 (Fig. 15) zu erwähnen, die an die qaabs in Umm el-Qaab erinnern, welche dort in Massen beim Osirisgrab geopfert wurden. ${ }^{96}$ Eine große Zahl dieser charakteristischen, grob gefertigten Kelche mit Brandspuren und Rückständen von Weihrauch und anderem Räucherwerk fand sich im vorderen Bereich der unterirdischen Anlage, v.a. in Raum 2 sowie im Lichthof ${ }^{97}$ Eine ähnlich hohe Konzentration dieser speziellen Keramik wurde auch im Grab des Harwa gefunden. Der Ausgräber der Anlage, F. Tiradritti, möchte dies mit den Bezügen von TT 37 auf das imaginäre Osirisgrab in Zusammenhang bringen und den Bau in ptolemäischer Zeit als Kultplatz erklären. ${ }^{98}$ Wie der Befund in TT 414 nahelegt, schlieBen sich Grab und Kultplatz zu dieser Zeit aber auf keinen Fall aus. Das Grab des Anch-Hor war in ptolemäischer Zeit sowohl ein Bestattungs-

96 Für eine Typologie der qaab-Opferschalen aus Abydos s. Müller 2003, 100-102 mit Abb. 11; bei der laufenden Freilegung des Osirisgrabes durch das DAI Kairo kommen weitere Kelche zum Vorschein, die denjenigen im Asasif genau entsprechen (persönliche Beobachtung im Herbst 2007).

$97 \mathrm{Zu}$ in situ-Funden in Raum $2 \mathrm{~s}$. BIETAK \& REISER-HASLAUER 1978, 85-88, Abb. 19-20; zur Keramik im Lichthof s. BietaK \& Reiser-Haslauer 1978, 150-151, Abb. 63.

98 S. Aston 2003, 162; Strudwick 2003, 174 mit Anm. 77 sowie TiradritTI 2005b, 170: „So the tomb of Harwa seems to have been transformed into a sanctuary during the Ptolemaic period. That idea is mainly derived from the study of the pottery. A first examination of the sherds recovered during excavations in the past seasons demonstrated a high concentration of votive cups and bowls dating to the Ptolemaic Period." 

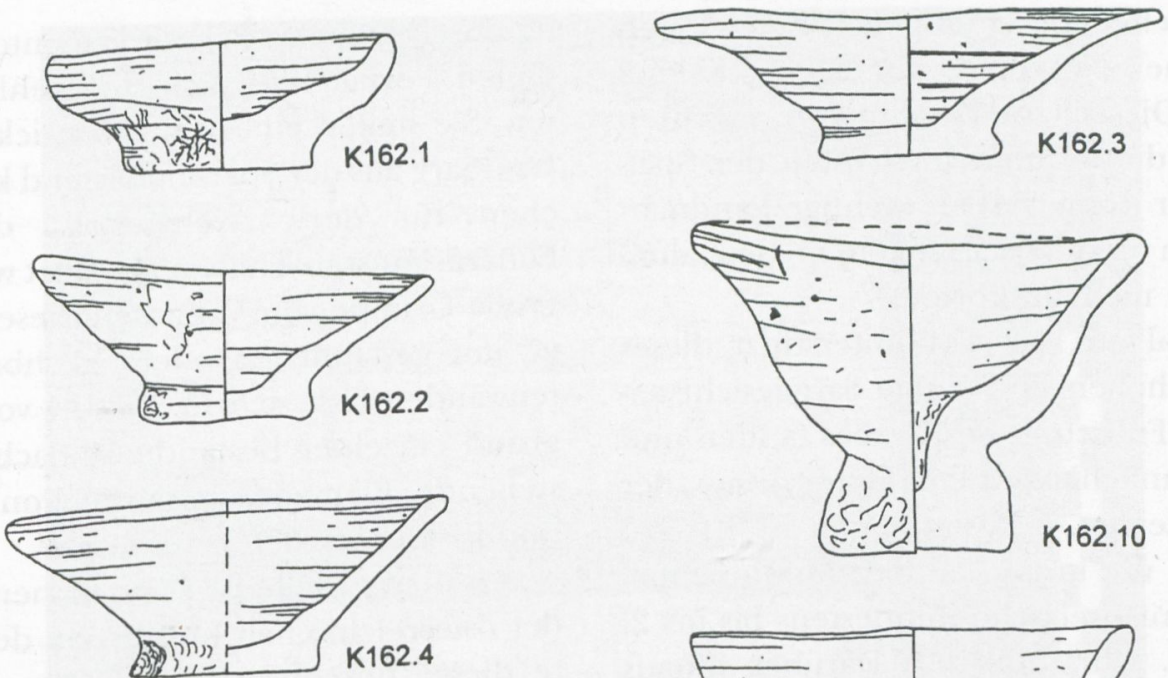

K162.10
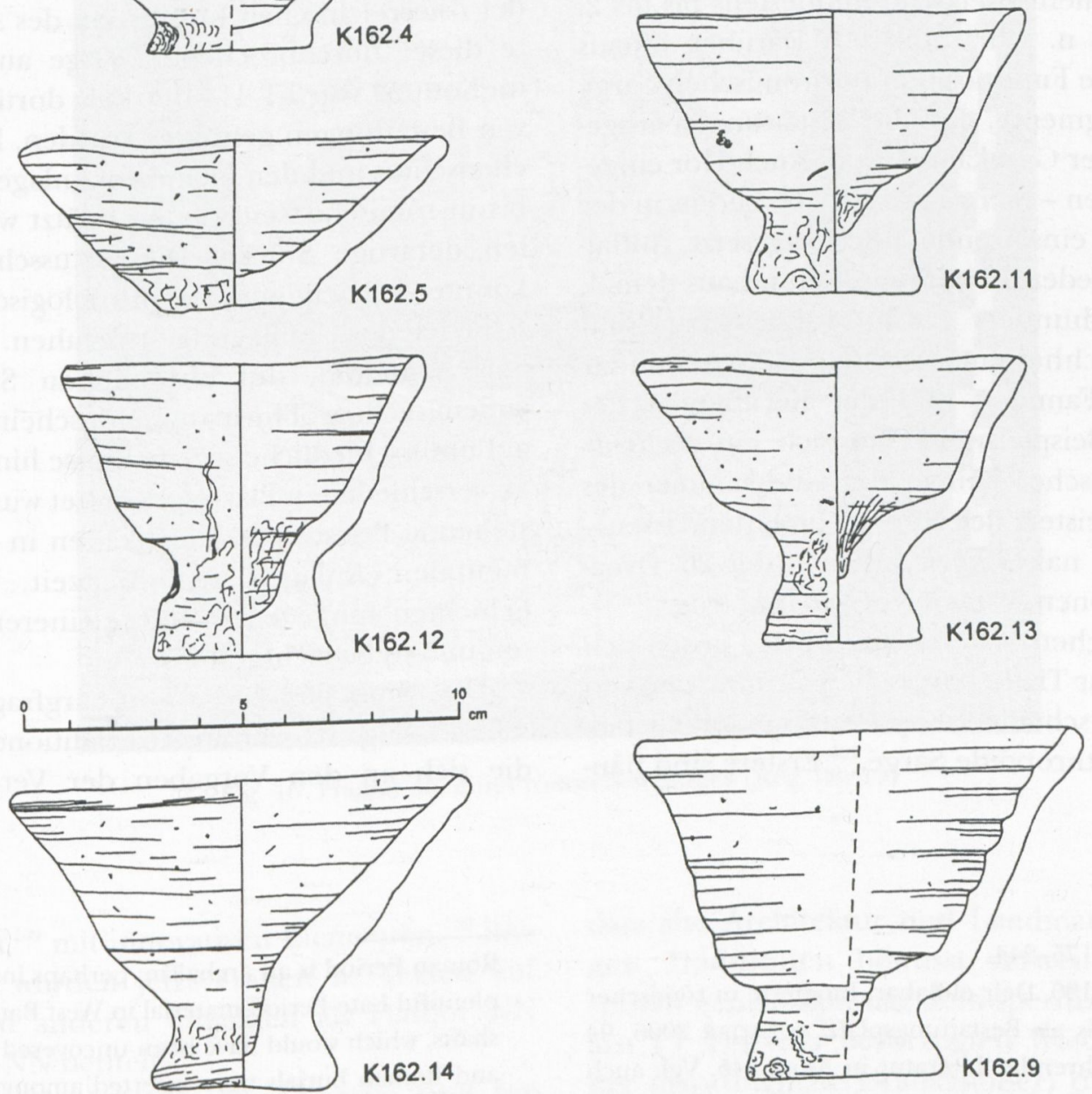

Fig. 15 Opferkeramik (Kelche und Modellschalen) aus TT 414, Raum 2 (4./3. Jh. v. Chr.)

(1:2, Zeichnungen: Zs. Thót)

platz als auch ein Ort, wo Totenritual und Festtagsopfer, beide in Zusammenhang mit dem Osiriskult, zelebriert wurden.

\section{Fundmaterial aus römischer Zeit}

Erst in jüngster Zeit hat die Erforschung der thebanischen Nekropole zu römischer Zeit deutliche Fortschritte verbuchen können. Nigel Strudwick und Christina Riggs haben die späte Entwicklung der thebanischen Friedhöfe, wobei das Asasif noch immer eine zentrale Rolle einnimmt, untersucht. ${ }^{99}$ Riggs hat außerdem 2005 eine Monogra-

99 Strudwick 2003; Riggs 2003. 
phie zu römischen Bestattungen vorgelegt und der thebanischen Nekropole ein eigenes Kapitel gewidmet. ${ }^{100}$ Diese Untersuchungen machten deutlich, dass die Monumentalbauten der Spätzeit auch in der Römerzeit als wichtige Landmarken fungierten und Bestattungen in den alten Vorzugsplätzen nicht aufhörten. ${ }^{101}$

Das Material aus TT 414 unterstützt dieses Bild. Hervorzuheben sind einige Sarggesichtsteile, die sich im Füllschutt des Grabes fanden und auffallende Ähnlichkeiten mit den Särgen der Soter-Familie zeigen. ${ }^{102}$ Bestattungen in TT 414 waren, ähnlich wie in den andern Monumentalgräbern, dementsprechend mindestens bis ins 2. Jahrhundert n. Chr. üblich. ${ }^{103}$ Darüber hinaus impliziert die Fundposition der römischen Särge und Sargfragmente, dass die Bestattungen ausgerechnet in der Grabkammer des Anch-Hor eingebracht wurden - hier wurde also die bereits in der 30. Dynastie einsetzende Sitte fortgesetzt. Auffallend, und wiederum mit dem Befund aus dem 4. und 3. Jahrhundert v. Chr. vergleichbar, sind Namensgleichheiten zwischen den sekundär bestatteten Familien und der Kernfamilie des Anch-Hor. Beispielsweise hat sich ein Ns-b3-nb$\underline{D} d . t$ in römischer Zeit in der Grabkammer des Obersthofmeisters der Nitokris bestatten lassen von dem ein naher Verwandter in der 26. Dynastie den schönen Namen Ns-b3-nb-Dd.t trug. ${ }^{104}$

Im römischen Material aus TT 414 lassen sich die beiden für Theben typischen Grundtypen von Särgen unterscheiden: Kapellensärge mit Giebeldach und anthropoide Särge. ${ }^{105}$ Erstere sind häu-

$\overline{{ }^{100} \text { RigGs 2005, } 175-244 .}$

${ }^{101}$ RigGs 2003, 190. Deir el-Bahari fungierte in römischer Zeit ebenfalls als Bestattungsplatz; s. ŁAJTAR 2006, 94 mit weiterführender Literatur in Anm. 345. Vgl. auch die ähnliche Beobachtung von MONTSERRAT 2000, 284 „elites continue to be buried in the same places as they had been for centuries, in spite of the democratisation of burial practices."

${ }^{102}$ Haslauer 2006.

${ }^{103}$ Im Grab des Padihorresnet kam ein ähnliches Sarggesichtsteil zum Vorschein, GRAEFE 2003, Bd. 1, 120; Bd. 2, Taf. 48, Kat. 27; im Grab des Harwa wurden zahlreiche Relikte römischen Grabinventars gefunden, darunter Glasphialen und Keramik, s. TIRADRITTI 2004b, 170, Abb. 6.

${ }^{104}$ S. Bietak \& Reiser-Haslauer 1982, G 99 und G 102.

${ }^{105}$ Riggs 2005, 186.

106 S. Riggs 2005, 186: „The revival of this form in the fig durch halbplastische Fragmente wie Pflanzensäulen, Portale und auch Hohlkehlen nachgewiesen. Sie stellen eine Weiterentwicklung des Typs krsw-Sarg aus der Spätzeit dar und können als Zeichen für den "Archaismus" der römische Funerärkunst in Theben gewertet werden. ${ }^{106}$ Eine große Gruppe von Varianten dieser Kapellensärge mit geschnitzten oder durchbrochenen Seitenwänden lässt sich in TT 414 vorwiegend aufgrund einzelner Bestandteile nachweisen - freistehende Elemente in Form von Djedpfeilern und Isisblut wurden gefunden. ${ }^{107}$

An dieser Stelle ist es erwähnenswert, dass in der österreichischen Konzession des Asasif Relikte dieser durchbrochenen Särge ausschließlich im Kontext von TT 414 und den dortigen intrusiven Bestattungen gefunden wurden. Im Oberflächenschutt und den kleineren Anlagen, die auch bis in römische Zeit wiederbenützt wurden, fehlen derartige Stücke. Diese Ausschließlichkeit könnte vielleicht auf feinchronologischen Unterschieden der Grablegungen beruhen. Das Fehlen von bemalten, durchbrochenen Särgen, die zudem recht großformatig sind, scheint aber eher auf unterschiedliche soziale Kreise hinweisen, die an verschiedenen Plätzen bestattet wurden: hochstehende Priesterpersönlichkeiten in den monumentalen Anlagen der Spätzeit, einfachere Schichten hingegen in den kleineren Grabbauten und in Schachtgräbern. ${ }^{108}$

Die römischen Särge und Sargfragmente aus TT 414 (Fig. 16) verbinden traditionelle Motive, die sich an den Vorgaben der Vergangenheit
Roman Period is an archaism, perhaps inspired by the plentiful Late Period material in West Bank tombs and shafts, which would have been uncovered as Ptolemaic and Roman burials were inserted among earlier burials." Für eine Liste römischer Pfostensärge s. auch BEINLICH-SEEBER 1998, 34, Anm. 87.

${ }^{107}$ Vgl. Bietak \& Reiser-Haslauer 1982, 155. Für vollständige Sargschreine s. ALtenMüller 1976, Taf. 6, unten; Riggs 2003, 193, Abb. 1. Auch im Tempelkontext gibt es vergleichbare Schreine mit freistehenden Holzelementen, siehe GREEN 1987, 18-20, Nr. 28-31 (datiert ins 6.-4. Jh. v. Chr.).

${ }^{108}$ Dies würde damit konform gehen, dass Haussärge auch in der Spätzeit keineswegs bei Durchschnitts-Bestattungen zu finden sind, sondern vielmehr Mittelstand und höhere Ränge markierten; vgl. TAYLOR 2001a, 238 sowie TAYLOR 2001b, 174. 


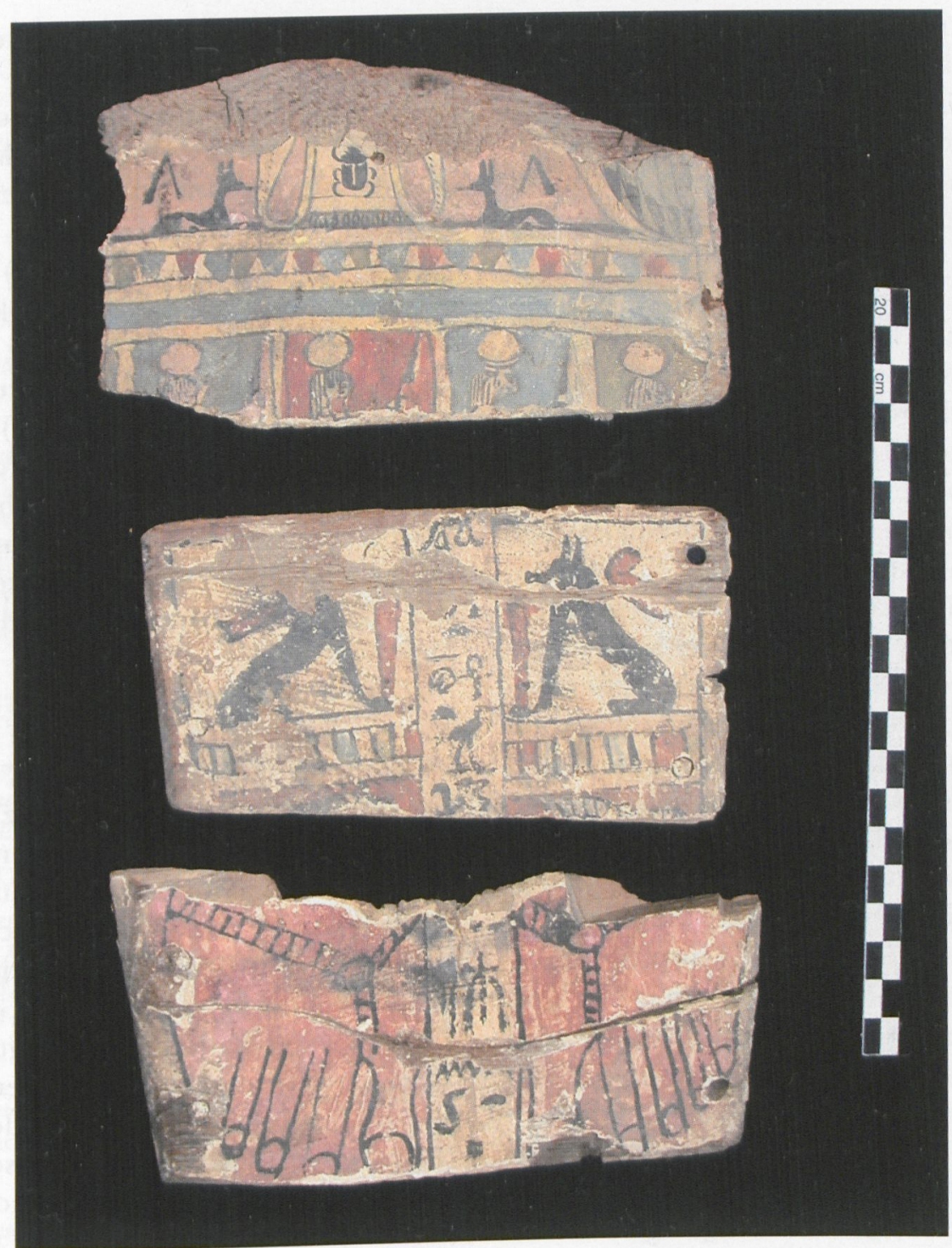

Fig. 16 Fragmente eines römischen Sarges (Reg. 08/11)

orientieren, ${ }^{109}$ mit innovativen Elementen. ${ }^{110}$ Beispielsweise wurden jetzt Frauen in Texten auf Särgen und anderen Objekten als Hathor NN statt Osiris NN betitelt. ${ }^{111}$

Ähnlich wie bei der 30. Dynastie sind wir momentan nicht in der Lage, den typisch thebanischen "Archaismus" und Konservatismus in römischer Zeit restlos zu erklären. Die Tatsache, dass alte Architektur und Landmarken mit langen Traditionen bewusst verwendet wurden, spricht gemeinsam mit dem spezifischen Befund aus TT 414 (der bevorzugten Wiederbenutzung der ursprüngliche Grabkammer) dafür, dass tatsächlich, wie oben auch schon für die 30. Dynastie vermutet wurde, eine Inspiration von den älteren Stücken ausgegangen ist. ${ }^{112}$ Ähnlich wie

\footnotetext{
${ }^{109}$ Zum konservativen Aspekt der römischen Funerärkunst s. RigGs 2005, 175; CORBELli 2006, 47.

${ }^{110}$ Vgl. RigGs 2005, 199.

${ }^{111}$ S. Verhoeven \& Witthuhn 2003, 311, Anm. $10 \mathrm{mit}$ weiterführender Literatur.

${ }^{112}$ Vgl. ähnlich auch Riggs 2005, 186: „The revival of this
}

form in the Roman Period is an archaism, perhaps inspired by the plentiful Late Period material in West Bank tombs and shafts, which would have been uncovered as Ptolemaic and Roman burials were inserted among earlier burials." 
bei dem sogenannten „Archaismus“ der 30. Dynastie gilt es hier aber zu differenzieren - wurden doch keine reinen Kopien und Nachahmungen angefertigt, sondern auf der Grundlage älterer Formen und Motive innovative neue Interpretationen geschaffen. ${ }^{113}$

\section{ZuSAMmenfassung UND AUSBlick}

Das Material aus TT 414, das momentan im Magazin der österreichischen Grabungen vor Ort im Asasif gelagert ist, stellt ein sehr großes Korpus an vielseitigen Gegenständen dar. Wegen seiner Provenienz aus einem geschlossenen Kontext mit einer zeitlichen Spannweite vom 6. Jahrhundert v. Chr. bis ins 2. Jahrhundert n. Chr. besitzt das Material enormes Potential für ein besseres Verständnis der späten Entwicklung des Bestattungsbrauchtums in der thebanischen Nekropole. Erste Studien gestatten interessante Einblicke in das vielseitige und lange Gebrauchsleben von TT 414. Eine wesentliche Erkenntnis dürfte sein, dass spätere Generationen bewusst einige Aspekte der ursprünglichen Gestaltung übernahmen, modifizierten und sich von Fundgegenständen inspirieren lieBen oder diese wiederverwendeten. Für ein genaues Verständnis dieser Beeinflussung sind noch weiterführende Studien notwendig. So könnten sich Einblicke in die Zusammenhänge der "Saitischen Renaissance“, die in der 30. Dynastie stattfand, ergeben. Die Innovationen der Ptolemäerzeit (vgl. den dargelegten Funktionswandel der Holzstelen) sowie die neuartigen Umsetzungen traditioneller Motive und Formen in der Römerzeit können ebenfalls gemeinsam mit dem älteren Material untersucht werden.

Im Anschluss an die 2007 und 2008 durchgeführte Inventur der Funde im Magazin sind nun gezielte Aufarbeitungskampagnen unter Einbeziehung von Spezialisten (Holz- und Papyrusrestauratoren, Anthropologen etc.) notwendig, um sowohl die wissenschaftliche Dokumentation als auch die fachgerechte Lagerung der Objekte zu garantieren. Als erster Schritt zur Leerung des provisorischen Magazins (Grab I) wurden vorerst vier fast vollständig erhaltene Särge und 50 weitere Objekte (Mumienbinden, Schrein- und Sargbretter, Statuetten, Uschebtis und Keramik) in eigens angefertigten Kisten ins zentrale Magazin der Ägyptischen Antikenbehörde beim CarterHaus überführt.

Die Fertigstellung einer dritten Monographie zum Grab des Anch-Hor, diesmal zu den Funden, ist durch die laufenden Arbeiten in unmittelbare Greifweite gerückt. Die Relevanz dieser Objekte geht trotz der starken Vermischung des Fundguts über die einer reinen Materialsammlung hinaus, da die gesamte Entwicklungsgeschichte der Blütezeit des Asasif, von der Saitenzeit bis in die römische Epoche, anhand der Gegenstände aus TT 414 exemplarisch aufgezeigt werden kann. Trotz jüngerer Studien ist dieser Abschnitt der ägyptischen Geschichte besonders in Theben noch immer unzureichend erschlossen - die Funde aus dem Grab des Anch-Hor können wesentlich dazu beitragen diese Lücke in der thebanischen Archäologie zu schließen und im speziellen die funeräre Kultur dieser Periode mit ihren zahlreichen Facetten zu fassen.

\footnotetext{
${ }^{113}$ Vgl. RigGs 2005, 244: „remains of earlier burials inspired archaism in new works of arts.“
} 


\section{Bibliographie}

AltenmülleR, $\mathrm{H}$.

1976 Grab und Totenreich der Alten Ägypter, Hamburg.

1977 s.v. Grabausstattung und -beigaben, LÄ II, 837-845.

ASSMANN, J.

1973 Das Grab des Basa (Nr. 389) in der Thebanischen Nekropole, AV 6, Mainz am Rhein.

1977 Das Grab der Mutirdis, AV 13, Mainz am Rhein.

Aston, D. A.

1987 Tomb Groups from the End of the New Kingdom to the Beginning of the Saite Period, unpublizierte Dissertation, University of Birmingham.

2003 The Theban West Bank from the Twenty-fifth Dynasty to the Ptolemaic Period, 138-166, in: N. STRUDWICK/J. H. TAYLOR 2003.

Bataille, A.

1951 Thèbes gréco-romaine, CdÉ 26, 325-353.

1952 Les Memnonia. Recherches de Papyrologie et d'épigraphie grecques sur la Nécropole de la Thèbes d'Égypte aux époques hellénistiques et romaines, RAPH 23.

BeINLICH-SEEBER, C.

1998 Ein römerzeitliches Sargfragment in Marseille, 9-40, in: A. BrodBeck (Hg.), Ein ägyptisches Glasperlenspiel. Ägyptologische Beiträge für Erik Hornung aus seinem Schülerkreis, Berlin.

Bierbrier, M. L.

1987 Hieroglyphic Texts from Egyptian Stelae etc., Part 11, BMP, London.

BIETAK, M.

1972 Theben-West (Luqsor). Vorbericht über die ersten vier Grabungskampagnen (1969-1971), SÖAW 278, 4, Wien.

1973 Ausgrabungen in Theben West - Asasif, AfO 24, 1973, 230-239.

1974-77 2. Bauforschungsprojekt: Die großen Saitengräber in der thebanischen Nekropole, AfO 25, 328-330.

1976-77 Theben-West (Vorbericht für 1973 und 1974), JbÖAI 51, Beiheft , 46-53.

Bietak, M. \& E. Reiser-Haslauer

1978 Das Grab des Anch-Hor, Obersthofmeister der Gottesgemahlin Nitokris I. Mit einem Beitrag von Erhart Graefe und Relief- und Fundzeichnungen von Heinz Satzinger, UZK 4, Wien.

1982 Das Grab des Anch-Hor, Obersthofmeister der Gottesgemahlin Nitokris II. Mit Beiträgen von Joachim Boessneck, Angela von den Driesch, Jan Quaegebeur, Helga Liese-Kleiber und Helmut Schlichtherle und Relief- und Fundzeichnungen von Heinz Satzinger, UZK 5, Wien.

BONOMI, J.

1906 Topographical Notes on Western Thebes Collected in $1830, A S A E 7,78-86$.
BUDKA, J.

2003 Ptah-Sokar-Osiris-Statuetten aus Grab VII im Asasif, 32-42, in: M. Hasitzka, J. Diethart \& G. Dembski (Hgg.), Das Alte Ägypten und seine Nachbarn, Festschrift zum 65. Geburtstag von Helmut Satzinger, Krems 2003.

2006 Die Spätzeit in Theben-West: Das Asasif. Bestattungsbrauchtum und Friedhofsstruktur anhand der Ergebnisse der österreichischen Ausgrabungen in den Jahren 1969-1977, unpublizierte Dissertation, Universität Wien.

Corbeli, J. A.

2006 The Art of Death in Graeco-Roman Egypt, Shire Egyptology 28, Princes Risborough.

EIGNER, D.

1984 Die monumentalen Grabbauten der Spätzeit in der Thebanischen Nekropole, UZK 6, Wien.

GOMAà, F.

2004 Bericht über die Freilegung des Grabes TT 197 des Padineith, Memnonia 15, 183-196.

2006 Die Arbeiten am Grab des Monthemhet, Sokar 12, 2006, 62-64.

GRAEFE, E.

1978 III. Stellung, Familie und Herkunft des 'Anch-Hor, 41-53, in: M. BietaK \& E. Reiser-Haslauer 1978.

1986 s.v. „Talfest“, in: LÄ VI, 187-189.

1990 Das Grab des Ibi, Obervermögenverwalter der Gottesgemahlin des Amun (Thebanisches Grab Nr. 36), Publication du Comité des Fouilles Belges en Egypte, Bruxelles.

2003 Das Grab des Padihorresnet, Obervermögensverwalter der Gottesgemahlin des Amun (Thebanisches Grab Nr. 196), Monumenta Aegyptiaca IX, Tournhout.

GRAJETZKI, W.

2003 Burial Customs in Ancient Egypt: Life in Death for Rich and Poor, London.

Green, C. I.

1987 The temple furniture from the Sacred Animal Necropolis at North Saqqara: 1964-1976, Excavations at North Saqqâra, Excavation Series, EES Excavation Memoir 53, London.

HAIKAL, F. M. H.

1970 Two hieratic funerary papyri of Nesmin, 2 Bde., Bibliotheca Aegyptiaca 14 und 15, Brüssel.

HASLAUER, E.

2006 Gesichter von Särgen aus dem Asasif. Eine Ergänzung zu den Särgen der Soter-Familie, 121-128, in: E. Czerny, I. Hein, H, Hunger, D. Melman \& A. SCHWAB (Hgg.), Timelines. Studies in honour of Manfred Bietak III, OLA 149.3, Leuven-Paris-Dudley, MA.

JANSEN-WINKELN, K.

1997 Eine Grabübernahme in der 30. Dynastie, JEA 83, 169-178. 
Kuhlmann, K.-P.

1973 Eine Beschreibung der Grabdekoration mit der Aufforderung zu kopieren und zum Hinterlassen von Besucherinschriften aus saitischer Zeit, MDAIK 29, 205-213.

Kuhlmann, K.-P. \& W. SCHENKEL

1983 Das Grab des Ibi, Obergutsverwalters des Amun, Band 1 (Text und Tafeln), AV 15, Mainz am Rhein.

ŁAJTAR, A.

2006 Deir el-Bahari in the Hellenistic and Roman Periods. A study of an Egyptian temple based on Greek sources, The Journal of Juristic Papyrology, Supplements, Vol. IV, Warsaw.

LANE, E. W.

2000 Description of Egypt, Notes and Views in Egypt and Nubia, Made During the Years 1825, -26, -27, and -28, hg. von J. THOMPSON, Kairo.

LEAHY, A.

1980 "Harwa" and "Harbes", CdÉ 55, 43-63.

LEPROHON, R.J.

1991 Stelae II, The New Kingdom to the Coptic Period, CAA 3, Mainz/Rhein.

LIPINSKA, J.

2008 An Unusual Wooden Statuette of Osiris, 166-169, in: S.H. D'Auria (Hg.), Servant of Mut. Studies in Honor of Richard A. Fazzini, $\operatorname{PdÄ~28,~Leiden-Boston.~}$

Lотн, M.

2008 "...painted funeral tablets of wood of the usual character..." Thebanische Totenstelen der 3. Zwischenzeit, Isched, Journal des Aegypten Forum Berlin e.V. 01/2008, 15-24.

Martin, C.J. \& K. RyHOLT

2006 Put my Funerary Papyrus in my Mummy, Please, JEA 92, 270-274.

MARTIN, G.T.

2005 Stelae from Egypt and Nubia in the Fitzwilliam Museum, Cambridge, c. 3000 BC-AD 1150, Cambridge 2005 .

De Meulenaere, $\mathrm{H}$.

1984 20. Une famille de «grands de la demeure de Khonsou", 238-241, in: M.L. Bierbrier, H. DE MeulenaeRE, S. SNAPE \& J.H. TAYLOR, Notes de prosopographie thébaine. Troisième série, $C d E ́$ 59, 199-241.

1989 Notes de prosopographie thébaine, Quatrième série, CdÉ 64, 1989, 55-73.

Millet, N. B.

2004 A Wooden Stela in the Royal Ontario Museum, 303- 306, in: G.N. KNOPPERS \& A. Hirsch (Hgg.), Egypt, Israel and the Ancient Mediterranean World. Studies in Honor of Donald. B. Redford, PdÄ 20, Leiden \& Boston.

MinAS-NERPEL, M.

2006 Der Gott Chepri: Untersuchungen zu Schriftzeugnissen und ikonographischen Quellen vom Alten Reich bis in griechisch-römische Zeit, OLA 154, Leuven-Dudley, Mass.

MONTSERRAT, D.

2000 Burial practices at Third Century A. D. Deir el Medina as evidenced from a Roman painted shroud in the Rijksmuseum van Oudheden, Leiden, 277-286, in: R. J. Demarée \& A. Egberts (Hgg.), Deir el Medina in the third millenium $A D$, EU 14, Leiden 2000.

MÜller, V.

2003 3. Deponierungen östlich und südöstlich des Grabes, 94-102, in: G. DreYer et al., Umm el-Qaab. Nachuntersuchungen im frühzeitlichen Königsfriedhof, 13./14./15. Vorbericht, MDAIK 59, $67-138$.

MunRo, P.

1973 Die spätägyptischen Totenstelen, ÄF 25, Glückstadt.

NIWINSKI, A.

1988 Sarcophagi, Stelae and Funerary Papyri of the Third Intermediate Period and the Late Period, 212-225, in: A.M. DONADONI Roveri (Hg.), Egyptian Civilization. Religious Beliefs, Egyptian Museum of Turin, Milano 1988.

Orton, C. \& Tyers, P. \& Vince, A.

1993 Pottery in Archaeology, Cambridge Manuals in Archaeology 1, Cambridge.

QUiBELl, J.E. \& GRIFFITH, F.LL.

1896 The Ramesseum Eo The Tomb of Ptah-Hetep, ERA 2, London, Neudruck 1989.

QUiRKE, S.

1993 Owners of Funerary Papyri in the British Museum, British Museum Occasional Paper 92, London.

RigGs, C.

2003 The Egyptian funerary tradition at Thebes in the Roman Period, 189-201, in: N. STRUdwick, J.H. TAYLOR (Hgg.), 2003.

2005 The Beautiful Burial in Roman Egypt. Art, Identity, and Funerary Religion, Oxford.

SATZINGER, $\mathrm{H}$.

1979 Theben, 95-114, in: Funde aus Ägypten. Österreichische Ausgrabungen seit 1961, Katalog KHM, Wien.

STRUDWICK, N.

2003 Some aspects of the archaeology of the Theban necropolis in the Ptolemaic and Roman periods, 167-188, in: N. Strudwick, J. H. TaYlor (Hgg.), 2003.

Strudwick, N. and J.H. TAYlor (Hgg.)

2003 The Theban Necropolis, Past, Present and Future, London.

TAYLOR, J.H.

1989 Egyptian Coffins, Shire Egyptology 11, Aylesbury.

2001a Death $\mathcal{E}^{\circ}$ the Afterlife in Ancient Egypt, London. 
2001b Patterns of colouring on ancient Egyptian coffins from the New Kingdom to the Twenty-Sixth Dynasty: an overview, 164-181, in: W.V. DAvies (Hg.), Colour and Painting in Ancient Egypt, London.

2003 Theban coffins from the Twenty-second to the Twenty-sixth Dynasty: dating and synthesis of development, 95-121, in: N. Strudwick, J. H. TAYLOR (Hgg.), 2003.

TIRADRITTI, F.

2004a Archaeological Activities of the Museum of Milan in the tomb of Harwa (TT 37) November-December 1996, ASAE 78, 161-166.

2004b Archaeological Activities of the Museum of Milan in the tomb of Harwa (TT 37) October-November1997, ASAE 78, 167-173.

2004c Archaeological Activities of the Museum of Milan in the tomb of Harwa (TT 37) September-November 1998, ASAE 78, 175-180.

2004d Archaeological Activities of the Museum of Milan in the tomb of Harwa (TT 37) 1999-2000, ASAE 78, 181-190.

2004e Archaeological Activities of the Museum of Milan in the tomb of Harwa (TT 37) 2000, ASAE 78, 191-197.

2005a Archaeological Activities of the Museum of Milan in the tomb of Harwa (TT 37) and Akhimenru (TT 404). April 2001, ASAE 79, 165-168.

2005b Archaeological Activities of the Museum of Milan in the tomb of Harwa (TT 37) and Akhimenru (TT 404). October-December 2001, ASAE 79, 169-178.

2005c Archaeological Activities of the Museum of Milan in the tomb of Harwa (TT 37) and Akhimenru (TT 404). October 2002-January 2003, ASAE 79, $179-186$.

2006 Italian Archaeological Mission to Luxor: Researches in the tombs of Harwa (TT 37) and Akhimenru (TT 404), ASAE 80, 563-569.

Traunecker, C., F. le Saout \& O. Masson

1981 La Chapelle d'Achôris à Karnak, Mémoires du Centre Franco-Égyptien d'Étude des Temples de Karnak, vol. 2, Paris.

Verhoeven, U. \& O. Witthuhn

2003 Eine Marburger Totenstele mit Anruf an die Lebenden, $S A K$ 31, 2003, 307-315.

\section{VitTMAnNN, G}

1978 Priester und Beamte im Theben der Spätzeit, BzÄ 1, Wien.

WIEBACH, S

1986 Die Begegnung von Lebenden und Verstorbenen im Rahmen des thebanischen Talfestes, SAK 13 , 264-291.

ZAYED, A. H.

1968 Painted wooden Stelae in the Cairo Museum, RdÉ 20, 149-170. 